\title{
NEAR-FIELD IMAGING OF SMALL PERTURBED OBSTACLES FOR ELASTIC WAVES
}

\author{
PEIJUN LI AND YULIANG WANG
}

\begin{abstract}
Consider an elastically rigid obstacle which is buried in a homogeneous and isotropic elastic background medium. The obstacle is illuminated by an arbitrary time-harmonic elastic incident wave. This work is concerned with an inverse obstacle scattering problem for elastic waves, which is to reconstruct the obstacle's surface from the displacement field measured at a circle surrounding it. The surface of the obstacle is assumed to be a small and smooth perturbation of a disk. Based on the Helmholtz decomposition, the displacement field is split into its compressional and shear parts. A coupled system of boundary value problems is derived for the decomposed scalar potentials by using the transparent boundary conditions. Utilizing the method of the transformed field expansion, we reduce the coupled system into to a successive sequence of one-dimensional twopoint boundary value problems which are solved in closed forms. The inverse problem is linearized by dropping high order terms in the power series expansion and an explicit reconstruction formula is obtained. Moreover, a simple nonlinear correction algorithm is proposed to improve the accuracy of the reconstructions. Ample numerical examples show that the method is capable of reconstructing the surface with subwavelength resolution.
\end{abstract}

\section{INTRODUCTION}

Wave imaging refers to the technology of determining geometrical or physical properties of an object by using acoustic, electromagnetic, or elastic waves. It has significant applications in broad scientific areas such as seismic tomography, non-destructive testing, and medical imaging. The underlying mathematical problems are known as the inverse scattering problems, which have been an active research area for decades.

This paper is concerned with near-field imaging of obstacles by using elastic waves. Here, nearfield indicates that the measurement is taken at a subwavelength distance from the object, while far-field means that the measurement is taken at a distance which is comparable with or longer than the wavelength. Due to the loss of high frequency information contained in the rapidly decaying wave components, the achievable resolution by far-field imaging modalities is limited to about one half of the wavelength, which is referred to as the Rayleigh criterion or the diffraction limit [11]. Near-field imaging is an effective approach to breaking the limit and obtaining super-resolution [15]. We refer to $[4,9,14,16-18]$ on mathematical and numerical studies for far-field imaging of obstacles with elastic waves. The linear sampling method was adopted in [13] for the reconstruction of obstacles buried in a semi-infinite domain using elastic waves.

Recently, a novel approach has been developed to solve a class of inverse surface scattering problems for acoustic and electromagnetic waves, which include infinite rough surfaces [6,7], diffraction gratings $[5,8,10]$, obstacles [20], and interior cavities [21]. The approach was also applied successfully in [23] to the inverse scattering of periodic surfaces for elastic waves. The goal of this work is to investigate the method on solving the inverse obstacle scattering problem for elastic waves. The new ingredients include convolution of Fourier coefficients and cylindrical functions. Due to the coupling of pressure and shear wave components traveling at different speeds, the governing Navier equation for elastic waves has a richer structure than the Helmholtz equation for acoustic waves and Maxwell's equations for electromagnetic waves, which makes the calculations much more

Key words and phrases. inverse obstacle scattering, elastic waves, near-field imaging, transformed field expansion, super-resolution.

The research was supported in part by the NSF grant DMS-1151308. 
sophisticated. Consequently, this paper is a nontrivial extension of the previous works because of the new challenges.

Specifically, we consider a two-dimensional impenetrable obstacle, which is elastically rigid and immersed in an unbounded, homogeneous, and isotropic elastic background medium. The surface of the obstacle is assumed to be a small and smooth perturbation of a circle. The obstacle is illuminated by an arbitrary time-harmonic elastic wave, which includes the commonly used plane waves, point sources, or a combination of both types. The displacement field of the total wave is measured on a circle near the surface. Using the Helmholtz decomposition, we split the displacement vector field into its compressional and shear parts by introducing two scalar potential functions. Transparent boundary conditions are derived for each part of the potential function through the Fourier series expansion. A coupled system of boundary value problems is deduced for the decomposed scalar potentials. Utilizing the transformed field expansion, which consists of a coordinate transformation, power series expansion, and the Fourier series expansion, we reduce the two-dimensional boundary value problem into a successive sequence of one-dimensional two-point boundary value problems, which are solved in closed forms. By neglecting the high order terms in the power series expansion, the inverse problem is linearized and formulated into an infinite dimensional linear system of equations relating the Fourier coefficients of the surface function and the data. An explicit reconstruction formula is obtained by solving the truncated linear system. Moreover, a simple nonlinear correction algorithm is developed to improve the accuracy of the reconstructions. The method requires a single incident field at a fixed frequency. It is computationally efficient and delivers excellent reconstructions with subwavelength resolution. We refer to [1-3] for related inverse elastic scattering problems and conductivity interface problems by using perturbation methods.

The outline of the paper is as follows. In section 2 , the model problem is formulated, transparent boundary conditions are presented, and a coupled system of boundary value problems is formulated. In section 3, the transformed field expansion is introduced and the boundary value problems are solved analytically. Section 4 is devoted to the derivation of the reconstruction formula and a nonlinear correction algorithm. Numerical experiments are reported in section 5. The paper is concluded in section 6 with general remarks and directions for future research.

\section{Problem formulation}

In this section, we introduce the mathematical model problem for elastic scattering by impenetrable obstacles.

2.1. Model problem. Consider a two-dimensional elastically rigid obstacle. Let the obstacle's surface be described by the curve (in the polar coordinates)

$$
\Gamma_{f}=\{(r, \theta): r=a+f(\theta), \theta \in[0,2 \pi]\},
$$

where $a>0$ is a constant and $f$ is a periodic function in $C^{2}([0,2 \pi])$ such that $a+f(\theta)>0$ for all $\theta \in[0,2 \pi]$. We assume that $f$ takes the form

$$
f(\theta)=\varepsilon g(\theta),
$$

where $\varepsilon>0$ is a sufficiently small constant and is called the surface deformation parameter. Suppose that the infinite exterior domain

$$
\Omega_{f}=\{(r, \theta): r>a+f(\theta), \theta \in[0,2 \pi]\}
$$

is occupied by a homogeneous and isotropic elastic medium with a unit mass density. Let

$$
\Gamma_{b}=\{(r, \theta): r=b, \theta \in[0,2 \pi]\}
$$

be a circle surrounding the obstacle, where $b=a+h \quad$ with $h>\max _{\theta \in[0,2 \pi]} f(\theta)$. Denote by

$$
\Omega=\{(r, \theta): a+f(\theta)<r<b, \theta \in[0,2 \pi]\}
$$

the bounded domain enclosed by $\Gamma_{f}$ and $\Gamma_{b}$. 
Let the obstacle be illuminated by a time-harmonic incident wave $\boldsymbol{u}_{\text {in }}$ with time dependence $e^{-\mathrm{i} \omega t}$, where $\omega>0$ is the angular frequency. We consider the most general incident wave which is only required to satisfy the two-dimensional homogeneous Navier equation:

$$
\left(\Delta^{*}+\omega^{2}\right) \boldsymbol{u}_{\text {in }}=0 \text { in } \Omega_{f}
$$

where $\Delta^{*}=\mu \Delta+(\lambda+\mu) \nabla \nabla \cdot, \lambda$ and $\mu$ are the two Lamé constants satisfying $\mu>0$ and $\lambda+\mu>0$. For instance, the incident field can be a plane wave, a radiating wave generated by a point source, or a combination of multiple plane waves and/or multiple point sources. It can consist of compressional wave, shear wave, or a mixture of both types of waves.

The displacement vector field of the total wave $\boldsymbol{u}=\left[u_{1}, u_{2}\right]$ satisfies the same Navier equation:

$$
\left(\Delta^{*}+\omega^{2}\right) \boldsymbol{u}=0 \quad \text { in } \Omega_{f} .
$$

Since the obstacle is elastically rigid, it holds the homogeneous Dirichlet boundary condition:

$$
\boldsymbol{u}=0 \text { on } \Gamma_{f} .
$$

Given the time-harmonic incident field $\boldsymbol{u}_{\text {in }}$, the direct problem is to determine the displacement field $\boldsymbol{u}$ in $\Omega_{f}$ for a known scattering surface $\Gamma_{f}$. This paper is focused on the inverse problem: to reconstruct the scattering surface $\Gamma_{f}$ from the measurement of the displacement field on $\Gamma_{b}$. In particular, we are interested in the inverse problem in the near-field regime where the measurement distance $h=b-a$ is much shorter than the wavelength.

2.2. Transparent boundary conditions. To reduce the problem from the unbounded domain $\Omega_{f}$ to the bounded domain $\Omega$, we introduce a transparent boundary condition on $\Gamma_{b}$.

The total field $\boldsymbol{u}$ may be decomposed into the incident field $\boldsymbol{u}_{\text {in }}$ and the scattered field $\boldsymbol{u}_{\text {sc }}$, i.e., $\boldsymbol{u}=\boldsymbol{u}_{\text {in }}+\boldsymbol{u}_{\mathrm{sc}}$. Subtracting (2.2) from (2.3) yields the Navier equation for the scattered field:

$$
\left(\Delta^{*}+\omega^{2}\right) \boldsymbol{u}_{\mathrm{sc}}=0 \quad \text { in } \Omega_{f}
$$

We introduce the Helmholtz decomposition for the scattered field

$$
\boldsymbol{u}_{\mathrm{sc}}=\nabla \phi_{\mathrm{sc}}+\operatorname{curl} \psi_{\mathrm{sc}},
$$

where $\phi_{\text {sc }}$ and $\psi_{\text {sc }}$ are the compressional and shear scalar potential functions, respectively, and the vector curl operator is defined as $\operatorname{curl} v=\left[\partial_{y} v,-\partial_{x} v\right]$ for any scalar function $v$.

Substituting (2.5) into (2.4) yields

$$
\nabla\left[(\lambda+2 \mu) \Delta \phi_{\mathrm{sc}}+\omega^{2} \phi_{\mathrm{sc}}\right]+\operatorname{curl}\left[\mu \Delta \psi_{\mathrm{sc}}+\omega^{2} \psi_{\mathrm{sc}}\right]=0 .
$$

The above equation is fulfilled if $\phi_{\mathrm{sc}}$ and $\psi_{\mathrm{sc}}$ satisfy the homogeneous Helmholtz equations:

$$
\left(\Delta+\kappa_{\mathrm{p}}^{2}\right) \phi_{\mathrm{sc}}=0, \quad\left(\Delta+\kappa_{\mathrm{s}}^{2}\right) \psi_{\mathrm{sc}}=0,
$$

where $\kappa_{\mathrm{p}}=\omega / \sqrt{\lambda+2 \mu}$ and $\kappa_{\mathrm{S}}=\omega / \sqrt{\mu}$ are the compressional and shear wavenumbers, respectively.

Since $\phi_{\mathrm{sc}}$ and $\psi_{\mathrm{sc}}$ are periodic functions in $\theta$ with period $2 \pi$, the solutions of (2.6) admit the Fourier series expansions:

$$
\phi_{\mathrm{sc}}(r, \theta)=\sum_{n \in \mathbb{Z}} \phi_{\mathrm{sc}}^{(n)}(r) e^{\mathrm{i} n \theta}, \quad \psi_{\mathrm{sc}}(r, \theta)=\sum_{n \in \mathbb{Z}} \psi_{\mathrm{sc}}^{(n)}(r) e^{\mathrm{i} n \theta}, \quad r \geq b .
$$

Substituting (2.7) into (2.6) and noting $\Delta=\partial_{r r}+r^{-1} \partial_{r}+r^{-2} \partial_{\theta \theta}$ yields the Bessel equations:

$$
\left\{\begin{array}{l}
{\left[\partial_{r r}+r^{-1} \partial_{y}+\left(\kappa_{\mathrm{p}}^{2}-n^{2} r^{-2}\right)\right] \phi_{\mathrm{sc}}^{(n)}(r)=0,} \\
{\left[\partial_{r r}+r^{-1} \partial_{y}+\left(\kappa_{\mathrm{s}}^{2}-n^{2} r^{-2}\right)\right] \psi_{\mathrm{sc}}^{(n)}(r)=0 .}
\end{array}\right.
$$

The scattered fields are assumed to consist of only outgoing waves. Hence the solutions of (2.8) can be written as

$$
\phi_{\mathrm{sc}}^{(n)}(r)=\alpha_{n} H_{n}^{(1)}\left(\kappa_{\mathrm{p}} r\right), \quad \psi_{\mathrm{sc}}^{(n)}(r)=\beta_{n} H_{n}^{(1)}\left(\kappa_{\mathrm{s}} r\right),
$$

where $\alpha_{n}, \beta_{n} \in \mathbb{C}$, and $H_{n}^{(1)}$ is the Hankel function of the first kind with order $n$. 
Plugging (2.9) into (2.7) yields

$$
\phi_{\mathrm{sc}}(r, \theta)=\sum_{n \in \mathbb{Z}} \alpha_{n} H_{n}^{(1)}\left(\kappa_{\mathrm{p}} r\right) e^{\mathrm{i} n \theta}, \quad \psi_{\mathrm{sc}}(r, \theta)=\sum_{n \in \mathbb{Z}} \beta_{n} H_{n}^{(1)}\left(\kappa_{\mathrm{s}} r\right) e^{\mathrm{i} n \theta}, \quad r \geq b .
$$

Taking the partial derivatives with respect to $r$ on both sides of $(2.10)$ and evaluating at $r=b$ yields

$$
\partial_{r} \phi_{\mathrm{sc}}(b, \theta)=\sum_{n \in \mathbb{Z}} \kappa_{\mathrm{p}} \alpha_{n} H_{n}^{(1)^{\prime}}\left(\kappa_{\mathrm{p}} b\right) e^{\mathrm{i} n \theta}, \quad \partial_{r} \psi_{\mathrm{sc}}(b, \theta)=\sum_{n \in \mathbb{Z}} \kappa_{\mathrm{s}} \beta_{n} H_{n}^{(1)^{\prime}}\left(\kappa_{\mathrm{s}} b\right) e^{\mathrm{i} n \theta} .
$$

Evaluating (2.10) at $r=b$ and comparing with (2.11), we derive the transparent boundary conditions for the scattered potentials on $\Gamma_{b}$ :

$$
\left(\partial_{r}-\mathscr{T}_{\mathrm{p}}\right) \phi_{\mathrm{sc}}=0, \quad\left(\partial_{r}-\mathscr{T}_{\mathrm{s}}\right) \psi_{\mathrm{sc}}=0,
$$

where the boundary operators $\mathscr{T}_{\mathrm{p}}$ and $\mathscr{T}_{\mathrm{s}}$ are defined by

$$
\mathscr{T}_{\mathrm{p}} v=\sum_{n \in \mathbb{Z}} \gamma_{\mathrm{p}}^{(n)} v^{(n)} e^{\mathrm{i} n \theta}, \quad \mathscr{T}_{\mathrm{s}} v=\sum_{n \in \mathbb{Z}} \gamma_{\mathrm{s}}^{(n)} v^{(n)} e^{\mathrm{i} n \theta}
$$

Here $v^{(n)}$ are the Fourier coefficients of $v$ and

$$
\gamma_{\mathrm{p}}^{(n)}=\kappa_{\mathrm{p}} H_{n}^{(1)^{\prime}}\left(\kappa_{\mathrm{p}} b\right) / H_{n}^{(1)}\left(\kappa_{\mathrm{p}} b\right), \quad \gamma_{\mathrm{s}}^{(n)}=\kappa_{\mathrm{s}} H_{n}^{(1)^{\prime}}\left(\kappa_{\mathrm{s}} b\right) / H_{n}^{(1)}\left(\kappa_{\mathrm{s}} b\right) .
$$

Similarly, we can apply the Helmholtz decomposition on the incident field:

$$
\boldsymbol{u}_{\text {in }}=\nabla \phi_{\text {in }}+\operatorname{curl} \psi_{\text {in }},
$$

where $\phi_{\text {in }}$ and $\psi_{\text {in }}$ also satisfy the Helmholtz equations (2.6). Since $\phi_{\text {in }}$ and $\psi_{\text {in }}$ are periodic functions with period $2 \pi$, they admit the Fourier series expansions:

$$
\left\{\begin{array}{l}
\phi_{\mathrm{in}}(r, \theta)=\sum_{n \in \mathbb{Z}}\left[\alpha_{n}^{(1)} H_{n}^{(1)}\left(\kappa_{\mathrm{p}} r\right)+\alpha_{n}^{(2)} H_{n}^{(2)}\left(\kappa_{\mathrm{p}} r\right)\right] e^{\mathrm{i} n \theta}, \\
\psi_{\mathrm{in}}(r, \theta)=\sum_{n \in \mathbb{Z}}\left[\beta_{n}^{(1)} H_{n}^{(1)}\left(\kappa_{\mathrm{s}} r\right)+\beta_{n}^{(2)} H_{n}^{(2)}\left(\kappa_{\mathrm{s}} r\right)\right] e^{\mathrm{i} n \theta},
\end{array}\right.
$$

where $\alpha_{n}^{(1)}, \alpha_{n}^{(2)}, \beta_{n}^{(1)}, \beta_{n}^{(2)}$ are coefficients that can be determined once the incident field is specified, and $H_{n}^{(2)}$ is the Hankel function of the second kind with order $n$.

Combining (2.14) and (2.13), we get

$$
\begin{aligned}
\left(\partial_{r}-\mathscr{T}_{\mathrm{p}}\right) \phi_{\text {in }}= & \sum_{n \in \mathbb{Z}}\left\{\alpha_{n}^{(1)} \kappa_{\mathrm{p}} H_{n}^{(1)^{\prime}}\left(\kappa_{\mathrm{p}} b\right)+\alpha_{n}^{(2)} \kappa_{\mathrm{p}} H_{n}^{(2)^{\prime}}\left(\kappa_{\mathrm{p}} b\right)\right. \\
& \left.\quad-\gamma_{\mathrm{p}}^{(n)}\left[\alpha_{n}^{(1)} H_{n}^{(1)}\left(\kappa_{\mathrm{p}} b\right)+\alpha_{n}^{(2)} H_{n}^{(2)}\left(\kappa_{\mathrm{p}} b\right)\right]\right\} e^{\mathrm{i} n \theta}, \\
= & \sum_{n \in \mathbb{Z}} \alpha_{n}^{(2)} \kappa_{\mathrm{p}}\left[H_{n}^{(2)^{\prime}}\left(\kappa_{\mathrm{p}} b\right)-H_{n}^{(1)^{\prime}}\left(\kappa_{\mathrm{p}} b\right) H_{n}^{(2)}\left(\kappa_{\mathrm{p}} b\right) / H_{n}^{(1)}\left(\kappa_{\mathrm{p}} b\right)\right] e^{\mathrm{i} n \theta} \\
= & \sum_{n \in \mathbb{Z}}\left[\alpha_{n}^{(2)} \kappa_{\mathrm{p}} W\left(\kappa_{\mathrm{p}} b\right) / H_{n}^{(1)}\left(\kappa_{\mathrm{p}} b\right)\right] e^{\mathrm{i} n \theta}=\sum_{n \in \mathbb{Z}}\left[\alpha_{n}^{(2)} W(b) / H_{n}^{(1)}\left(\kappa_{\mathrm{p}} b\right)\right] e^{\mathrm{i} n \theta \quad \text { on } \Gamma_{b},}
\end{aligned}
$$

where $W$ is the Wronskian of $H_{n}^{(1)}$ and $H_{n}^{(2)}$ defined by

$$
W(z)=\left|\begin{array}{ll}
H_{n}^{(1)}(z) & H_{n}^{(2)}(z) \\
H_{n}^{(1)^{\prime}}(z) & H_{n}^{(2)^{\prime}}(z)
\end{array}\right|=-\left(\frac{4 \mathrm{i}}{\pi z}\right) .
$$

Denote

$$
\rho_{\mathrm{p}}=\sum_{n \in \mathbb{Z}}\left[\alpha_{n}^{(2)} W(b) / H_{n}^{(1)}\left(\kappa_{\mathrm{p}} b\right)\right] e^{\mathrm{i} n \theta}, \quad \rho_{\mathrm{s}}=\sum_{n \in \mathbb{Z}}\left[\beta_{n}^{(2)} W(b) / H_{n}^{(1)}\left(\kappa_{\mathrm{s}} b\right)\right] e^{\mathrm{i} n \theta} .
$$

It can be verified that

$$
\left(\partial_{r}-\mathscr{T}_{\mathrm{p}}\right) \phi_{\mathrm{in}}=\rho_{\mathrm{p}}, \quad\left(\partial_{r}-\mathscr{T}_{\mathrm{s}}\right) \psi_{\text {in }}=\rho_{\mathrm{s}} \quad \text { on } \Gamma_{b}
$$


Let the total wave $\boldsymbol{u}$ admit the following Helmholtz decomposition:

$$
\boldsymbol{u}=\nabla \phi+\operatorname{curl} \psi \text {. }
$$

Adding (2.12) and (2.17) yields the transparent boundary conditions for the scalar potentials of $\phi$ and $\psi$ :

$$
\left(\partial_{r}-\mathscr{T}_{\mathrm{p}}\right) \phi=\rho_{\mathrm{p}}, \quad\left(\partial_{r}-\mathscr{T}_{\mathrm{s}}\right) \psi=\rho_{\mathrm{s}} \quad \text { on } \Gamma_{b} .
$$

Next we restate the boundary condition on $\Gamma_{f}$ in the polar coordinate. It is easy to verify the differentiation rules:

$$
\partial_{x}=(\cos \theta) \partial_{r}-r^{-1}(\sin \theta) \partial_{\theta}, \quad \partial_{y}=(\sin \theta) \partial_{r}+r^{-1}(\cos \theta) \partial_{\theta}
$$

Substituting (2.20) to (2.18) and noting $\boldsymbol{u}=0$ on $\Gamma_{f}$ yields

$$
\left\{\begin{array}{l}
u_{1}=\left[(\cos \theta) \partial_{r}-r^{-1}(\sin \theta) \partial_{\theta}\right] \phi+\left[(\sin \theta) \partial_{r}+r^{-1}(\cos \theta) \partial_{\theta}\right] \psi=0 \\
u_{2}=\left[(\sin \theta) \partial_{r}+r^{-1}(\cos \theta) \partial_{\theta}\right] \phi-\left[(\cos \theta) \partial_{r}-r^{-1}(\sin \theta) \partial_{\theta}\right] \psi=0
\end{array}\right.
$$

Recall that a vector $\left[u_{1}, u_{2}\right]=0$ if and only if its projection to the orthonormal vectors $\hat{r}=$ $[\cos \theta, \sin \theta]$ and $\hat{\theta}=[-\sin \theta, \cos \theta]$ are zero, i.e.,

$$
u_{1} \cos \theta+u_{2} \sin \theta=0, \quad u_{1}(-\sin \theta)+u_{2}(\cos \theta)=0 .
$$

Substituting (2.21) into the above equations, we obtain the equivalent boundary conditions for the scalar potentials:

$$
r \partial_{r} \phi+\partial_{\theta} \psi=0, \quad r \partial_{r} \psi-\partial_{\theta} \phi=0 \quad \text { on } \Gamma_{f} .
$$

In summary, the scalar potentials $\phi$ and $\psi$ satisfy the coupled system of boundary value problems:

$$
\left\{\begin{array}{lll}
\left(\Delta+\kappa_{\mathrm{p}}^{2}\right) \phi=0, & \left(\Delta+\kappa_{\mathrm{s}}^{2}\right) \psi=0 & \text { in } \Omega, \\
r \partial_{r} \phi+\partial_{\theta} \psi=0, & r \partial_{r} \psi-\partial_{\theta} \phi=0 & \text { on } \Gamma_{f}, \\
\left(\partial_{r}-\mathscr{T}_{\mathrm{p}}\right) \phi=\rho_{\mathrm{p}}, & \left(\partial_{r}-\mathscr{T}_{\mathrm{s}}\right) \psi=\rho_{\mathrm{s}} & \text { on } \Gamma_{b} .
\end{array}\right.
$$

It is easy to note that $\phi$ and $\psi$ are decoupled in the equations over $\Omega$ and in the transparent boundary conditions on $\Gamma_{b}$ but they are coupled in the boundary conditions on $\Gamma_{f}$.

\section{Transformed Field Expansion}

In this section we derive an analytical solution for the coupled system of boundary value problems (2.23) using the technique of transformed field expansion. It consists of three steps: change of variables, power series expansion, and Fourier series expansion.

3.1. Change of variables. Consider the change of variables:

$$
\tilde{r}=\frac{h r-b f(\theta)}{h-f(\theta)}, \quad \tilde{\theta}=\theta,
$$

which transforms the domain $\Omega$ to the annulus $D=\{(r, \theta): a<r<b, \theta \in[0,2 \pi]\}$ by mapping the boundary $\Gamma_{f}$ to the circle $\Gamma_{a}=\{(r, \theta): r=a, \theta \in[0,2 \pi]\}$ while keeping the boundary $\Gamma_{b}$ unchanged.

We seek to restate the boundary value problems (2.23) in the new variables. Direct calculations yield the following differentiation rules:

$$
\begin{aligned}
\partial_{r} & =\left(\frac{h}{h-f}\right) \partial_{\tilde{r}}, \quad \partial_{\theta}=\partial_{\tilde{\theta}}+f^{\prime}\left(\frac{\tilde{r}-b}{h-f}\right) \partial_{\tilde{r}}, \quad \partial_{r r}=\left(\frac{h}{h-f}\right)^{2} \partial_{\tilde{r} \tilde{r}}, \\
\partial_{\theta \theta} & =\partial_{\tilde{\theta} \tilde{\theta}}+\left(f^{\prime}\right)^{2}\left(\frac{\tilde{r}-b}{h-f}\right)^{2} \partial_{\tilde{r} \tilde{r}}+2 f^{\prime}\left(\frac{\tilde{r}-b}{h-f}\right) \partial_{\tilde{\theta} \tilde{r}}+\left[f^{\prime \prime}\left(\frac{\tilde{r}-b}{h-f}\right)+2\left(f^{\prime}\right)^{2} \frac{(\tilde{r}-b)}{(h-f)^{2}}\right] \partial_{\tilde{r}}
\end{aligned}
$$


Introduce new functions $\tilde{\phi}(\tilde{r}, \tilde{\theta})=\phi(r, \theta)$ and $\tilde{\psi}(\tilde{r}, \tilde{\theta})=\psi(r, \theta)$ under the transformation. It can be verified after a tedious but straightforward calculation that the new functions, upon dropping the tilde on all variables for simplicity of notation, satisfy the following equations in $D$ :

$$
\left\{\begin{array}{l}
\left(c_{1} \partial_{r r}+c_{2} \partial_{r}+c_{3} \partial_{\theta \theta}+c_{4} \partial_{r \theta}+c_{5} \kappa_{p}^{2}\right) \phi=0, \\
\left(c_{1} \partial_{r r}+c_{2} \partial_{r}+c_{3} \partial_{\theta \theta}+c_{4} \partial_{r \theta}+c_{5} \kappa_{s}^{2}\right) \psi=0,
\end{array}\right.
$$

where the variable coefficients are given by

$$
\left\{\begin{aligned}
c_{1}= & h^{2} r^{2}-2 h r(r-b) f+(r-b)^{2}\left[f^{2}+\left(f^{\prime}\right)^{2}\right], \\
c_{2}= & h^{2} r-h(2 r-b) f+h(r-b) f^{\prime \prime}+(r-b)\left[f^{2}+2\left(f^{\prime}\right)^{2}-f f^{\prime \prime}\right], \\
c_{3}= & h^{2}-2 h f+f^{2} \\
c_{4}= & 2 h(r-b) f^{\prime}-2(r-b) f f^{\prime} \\
c_{5}= & h^{2} r^{2}-2 h r(2 r-b) f+\left(6 r^{2}-6 r b+b^{2}\right) f^{2} \\
& \quad-2 h^{-1}(r-b)(2 r-b) f^{3}+h^{-2}(r-b)^{2} f^{4} .
\end{aligned}\right.
$$

Under the change of variables, the boundary conditions $(2.22)$ on $\Gamma_{a}$ become

$$
\left\{\begin{array}{l}
(a+f) \partial_{r} \phi+\left[\left(1-h^{-1} f\right) \partial_{\theta}-f^{\prime} \partial_{r}\right] \psi=0 \\
(a+f) \partial_{r} \psi-\left[\left(1-h^{-1} f\right) \partial_{\theta}-f^{\prime} \partial_{r}\right] \phi=0
\end{array}\right.
$$

and the transparent boundary conditions $(2.19)$ on $\Gamma_{b}$ reduce to

$$
\left\{\begin{array}{l}
\partial_{r} \phi=\left(1-h^{-1} f\right)\left(\mathscr{T}_{p} \phi+\rho_{p}\right), \\
\partial_{r} \psi=\left(1-h^{-1} f\right)\left(\mathscr{T}_{s} \psi+\rho_{s}\right) .
\end{array}\right.
$$

3.2. Power series expansion. Recalling the assumption of the surface function (2.1), we employ a boundary perturbation approach and consider formal expansions of $\phi$ and $\psi$ as power series of the surface deformation parameter $\varepsilon$ :

$$
\phi(r, \theta ; \varepsilon)=\sum_{k=0}^{\infty} \phi_{k}(r, \theta) \varepsilon^{k}, \quad \psi(r, \theta ; \varepsilon)=\sum_{k=0}^{\infty} \psi_{k}(r, \theta) \varepsilon^{k} .
$$

Substituting (2.1) into (3.2) and (3.5) into (3.1), we obtain a successive sequence of equations:

$$
\left(\Delta+\kappa_{\mathrm{p}}^{2}\right) \phi_{k}=v_{k}, \quad\left(\Delta+\kappa_{\mathrm{s}}^{2}\right) \psi_{k}=w_{k} \quad \text { in } D,
$$

where

$$
v_{k}=r^{-2} \sum_{j=1}^{4} \mathscr{D}_{j}\left(\kappa_{\mathrm{p}}\right) \phi_{k-j}, \quad w_{k}=r^{-2} \sum_{j=1}^{4} \mathscr{D}_{j}\left(\kappa_{\mathrm{S}}\right) \psi_{k-j} .
$$

Here the differential operators $\mathscr{D}_{j}$ are given by

$$
\begin{aligned}
& \mathscr{D}_{1}(\kappa)=h^{-1}\left\{2 r(r-b) g \partial_{r r}+\left[(2 r-b) g-(r-b) g^{\prime \prime}\right] \partial_{r}+2 g \partial_{\theta \theta}\right. \\
& \left.-2(r-b) g^{\prime} \partial_{r \theta}+2 r(2 r-b) g \kappa^{2}\right\}, \\
& \mathscr{D}_{2}(\kappa)=h^{-2}\left\{-(r-b)^{2}\left[g^{2}+\left(g^{\prime}\right)^{2}\right] \partial_{r r}-(r-b)\left[g^{2}+2\left(g^{\prime}\right)^{2}-g g^{\prime \prime}\right] \partial_{r}-g^{2} \partial_{\theta \theta}\right. \\
& \left.+2(r-b) g g^{\prime} \partial_{r \theta}-\left(6 r^{2}-6 r b+b^{2}\right) g^{2} \kappa^{2}\right\}, \\
& \mathscr{D}_{3}(\kappa)=2 h^{-3}(r-b)(2 r-b) g^{3} \kappa^{2} \text {, } \\
& \mathscr{D}_{4}(\kappa)=-h^{-4}(r-b)^{2} g^{4} \kappa^{2} .
\end{aligned}
$$

Plugging (2.1) into the boundary conditions (3.3) yields

$$
\partial_{r} \phi_{k}+a^{-1} \partial_{\theta} \psi_{k}=p_{k}, \quad \partial_{r} \psi_{k}-a^{-1} \partial_{\theta} \phi_{k}=q_{k} \quad \text { on } \Gamma_{a},
$$


where

$$
\left\{\begin{array}{l}
p_{k}=-a^{-1}\left[g\left(\partial_{r} \phi_{k-1}-h^{-1} \partial_{\theta} \psi_{k-1}\right)-g^{\prime} \partial_{r} \psi_{k-1}\right] \\
q_{k}=-a^{-1}\left[g\left(\partial_{r} \psi_{k-1}+h^{-1} \partial_{\theta} \phi_{k-1}\right)+g^{\prime} \partial_{r} \phi_{k-1}\right] .
\end{array}\right.
$$

Similarly, the the boundary conditions (3.4) reduce to

$$
\left(\partial_{r}-\mathscr{T}_{\mathrm{p}}\right) \phi_{k}=\sigma_{k}, \quad\left(\partial_{r}-\mathscr{T}_{\mathrm{s}}\right) \psi_{k}=\tau_{k} \quad \text { on } \Gamma_{b},
$$

where

$$
\left\{\begin{array} { l } 
{ \sigma _ { 0 } = \rho _ { \mathrm { p } } , \quad \sigma _ { 1 } = - h ^ { - 1 } g ( \mathscr { T } _ { \mathrm { p } } \phi _ { 0 } + \rho _ { \mathrm { p } } ) , } \\
{ \sigma _ { k } = - h ^ { - 1 } g \mathscr { T } _ { \mathrm { p } } \phi _ { k - 1 } , \quad k \geq 2 , }
\end{array} \quad \left\{\begin{array}{l}
\tau_{0}=\rho_{\mathrm{s}}, \quad \tau_{1}=-h^{-1} g\left(\mathscr{T}_{\mathrm{s}} \psi_{0}+\rho_{\mathrm{s}}\right), \\
\tau_{k}=-h^{-1} g \mathscr{T}_{\mathrm{s}} \psi_{k-1}, \quad k \geq 2 .
\end{array}\right.\right.
$$

In all the above recurrence relations, it is understood that $\phi_{k}=\psi_{k}=0$ for $k<0$. Note that the boundary value problems (3.6)-(3.9) for the current terms $\phi_{k}, \psi_{k}$ involve $v_{k}, w_{k}, p_{k}, q_{k}, \sigma_{k}, \tau_{k}$, which in turn depend on $\phi_{k-j}, \psi_{k-j}$ for $j=1, \ldots, 4$. Hence the boundary value problems (3.6)-(3.9) can be solved in a recursive manner starting from $k=0$.

3.3. Fourier series expansion. Since all the functions in the boundary value problems (3.6)-(3.9) are periodic in $\theta$ with period $2 \pi$, we may adopt the Fourier series expansion to reduce the twodimensional boundary value problems into one-dimensional two-point boundary value problems in the frequency domain:

$$
\begin{cases}{\left[\partial_{r r}+r^{-1} \partial_{r}+\left(\kappa_{\mathrm{p}}^{2}-n^{2} r^{-2}\right)\right] \phi_{k}^{(n)}=v_{k}^{(n)},} & a<r<b \\ \partial_{r} \phi_{k}^{(n)}=p_{k}^{(n)}-\mathrm{i} n a^{-1} \psi_{k}^{(n)}, & r=a \\ \left(\partial_{r}-\gamma_{\mathrm{p}}^{(n)}\right) \phi_{k}^{(n)}=\sigma_{k}^{(n)}, & r=b\end{cases}
$$

and

$$
\begin{cases}{\left[\partial_{r r}+r^{-1} \partial_{r}+\left(\kappa_{\mathrm{s}}^{2}-n^{2} r^{-2}\right)\right] \psi_{k}^{(n)}=w_{k}^{(n)},} & a<r<b \\ \partial_{r} \psi_{k}^{(n)}=q_{k}^{(n)}+\mathrm{i} n a^{-1} \phi_{k}^{(n)}, & r=a \\ \left(\partial_{r}-\gamma_{\mathrm{s}}^{(n)}\right) \psi_{k}^{(n)}=\tau_{k}^{(n)}, & r=b .\end{cases}
$$

Note that the above two boundary value problems are coupled in the boundary conditions at $r=a$. To solve the coupled system, we consider $\psi_{k}^{(n)}(a)$ and $\phi_{k}^{(n)}(a)$ as constants and find the analytical solutions for each boundary value problem. By evaluating the solutions at $r=a$, we obtain a linear system of algebraic equations for $\psi_{k}^{(n)}(a)$ and $\phi_{k}^{(n)}(a)$. We then solve this linear system and substitute the results back to the analytical form to obtain the final solutions.

A direct application of Lemma A.1 yields the solutions of (3.10) and (3.11):

$$
\left\{\begin{aligned}
\phi_{k}^{(n)}(r)=- & K^{(n)}\left(r, a ; \kappa_{\mathrm{p}}\right)\left[p_{k}^{(n)}-\mathrm{i} n a^{-1} \psi_{k}^{(n)}(a)\right] \\
& +K^{(n)}\left(r, b ; \kappa_{\mathrm{p}}\right) \sigma_{k}^{(n)}-\int_{a}^{b} K^{(n)}\left(r, s ; \kappa_{\mathrm{p}}\right) v_{k}^{(n)}(s) \mathrm{d} s \\
\psi_{k}^{(n)}(r)=- & K^{(n)}\left(r, a ; \kappa_{\mathrm{S}}\right)\left[q_{k}^{(n)}+\mathrm{i} n a^{-1} \phi_{k}^{(n)}(a)\right] \\
& +K^{(n)}\left(r, b ; \kappa_{\mathrm{S}}\right) \tau_{k}^{(n)}-\int_{a}^{b} K^{(n)}\left(r, s ; \kappa_{\mathrm{S}}\right) w_{k}^{(n)}(s) \mathrm{d} s
\end{aligned}\right.
$$

where $K^{(n)}$ is given by $(\mathrm{A} .2)$ and $\phi_{k}^{(n)}(a), \psi_{k}^{(n)}(a)$ are to be determined. Evaluating the above solutions at $r=a$ and noting that

$$
\left\{\begin{array}{l}
K^{(n)}(a, a, \kappa)=-H_{n}^{(1)}(\kappa a) /\left(\kappa H_{n}^{(1)^{\prime}}(\kappa a)\right), \\
K^{(n)}(a, b, \kappa)=-b H_{n}^{(1)}(\kappa b) /\left(\kappa a H_{n}^{(1)^{\prime}}(\kappa a)\right), \\
K^{(n)}(a, s, \kappa)=-s H_{n}^{(1)}(\kappa s) /\left(\kappa a H_{n}^{(1)^{\prime}}(\kappa a)\right),
\end{array}\right.
$$


we obtain

$$
\left\{\begin{array}{l}
\kappa_{\mathrm{p}} a H_{n}^{(1)^{\prime}}\left(\kappa_{\mathrm{p}} a\right) \phi_{k}^{(n)}(a)+\mathrm{i} n H_{n}^{(1)}\left(\kappa_{\mathrm{p}} a\right) \psi_{k}^{(n)}(a)=\zeta_{k}^{(n)} \\
\kappa_{\mathrm{s}} a H_{n}^{(1)^{\prime}}\left(\kappa_{\mathrm{s}} a\right) \psi_{k}^{(n)}(a)-\mathrm{i} n H_{n}^{(1)}\left(\kappa_{\mathrm{s}} a\right) \phi_{k}^{(n)}(a)=\eta_{k}^{(n)}
\end{array}\right.
$$

where

$$
\left\{\begin{array}{l}
\zeta_{k}^{(n)}=a H_{n}^{(1)}\left(\kappa_{\mathrm{p}} a\right) p_{k}^{(n)}-b H_{n}^{(1)}\left(\kappa_{\mathrm{p}} b\right) \sigma_{k}^{(n)}+\int_{a}^{b} s H_{n}^{(1)}\left(\kappa_{\mathrm{p}} s\right) v_{k}^{(n)}(s) \mathrm{d} s \\
\eta_{k}^{(n)}=a H_{n}^{(1)}\left(\kappa_{\mathrm{s}} a\right) q_{k}^{(n)}-b H_{n}^{(1)}\left(\kappa_{\mathrm{s}} b\right) \tau_{k}^{(n)}+\int_{a}^{b} s H_{n}^{(1)}\left(\kappa_{\mathrm{s}} s\right) w_{k}^{(n)}(s) \mathrm{d} s
\end{array}\right.
$$

Hence we have a linear system of algebraic equations for $\phi_{k}^{(n)}(a)$ and $\psi_{k}^{(n)}(a)$ :

$$
\left[\begin{array}{cc}
\kappa_{\mathrm{p}} a H_{n}^{(1)^{\prime}}\left(\kappa_{\mathrm{p}} a\right) & \mathrm{i} n H_{n}^{(1)}\left(\kappa_{\mathrm{p}} a\right) \\
-\mathrm{i} n H_{n}^{(1)}\left(\kappa_{\mathrm{s}} a\right) & \kappa_{\mathrm{s}} a H_{n}^{(1)^{\prime}}\left(\kappa_{\mathrm{s}} a\right)
\end{array}\right]\left[\begin{array}{l}
\phi_{k}^{(n)}(a) \\
\psi_{k}^{(n)}(a)
\end{array}\right]=\left[\begin{array}{l}
\zeta_{k}^{(n)} \\
\eta_{k}^{(n)}
\end{array}\right] .
$$

A simple calculation from Cramer's rule yields its solution:

$$
\left\{\begin{array}{l}
\phi_{k}^{(n)}(a)=\Lambda_{n}^{-1}\left[\kappa_{\mathrm{s}} a H_{n}^{(1)^{\prime}}\left(\kappa_{\mathrm{s}} a\right) \zeta_{k}^{(n)}-\mathrm{i} n H_{n}^{(1)}\left(\kappa_{\mathrm{p}} a\right) \eta_{k}^{(n)}\right] \\
\psi_{k}^{(n)}(a)=\Lambda_{n}^{-1}\left[\kappa_{\mathrm{p}} a H_{n}^{(1)^{\prime}}\left(\kappa_{\mathrm{p}} a\right) \eta_{k}^{(n)}+\mathrm{i} n H_{n}^{(1)}\left(\kappa_{\mathrm{s}} a\right) \zeta_{k}^{(n)}\right]
\end{array}\right.
$$

where the determinant

$$
\Lambda_{n}=\kappa_{\mathrm{p}} \kappa_{\mathrm{s}} a^{2} H_{n}^{(1)^{\prime}}\left(\kappa_{\mathrm{p}} a\right) H_{n}^{(1)^{\prime}}\left(\kappa_{\mathrm{s}} a\right)-n^{2} H_{n}^{(1)}\left(\kappa_{\mathrm{p}} a\right) H_{n}^{(1)}\left(\kappa_{\mathrm{s}} a\right) .
$$

Plugging (3.14) into (3.12), we may obtain the solutions of $\phi_{k}^{(n)}(r)$ and $\psi_{k}^{(n)}(r)$ for all $k$ and $n$. Recalling the recurrence relations for Hankel functions

$$
H_{n}^{(1)^{\prime}}(z)=H_{n-1}^{(1)}(z)-(n / z) H_{n}^{(1)}(z), \quad(2 n / z) H_{n}^{(1)}(z)=H_{n-1}^{(1)}(z)+H_{n+1}^{(1)}(z),
$$

we may deduce the following alternative form for $\Lambda_{n}$ :

$$
\Lambda_{n}=-\frac{1}{2} \kappa_{\mathrm{p}} \kappa_{\mathrm{s}} a^{2}\left[H_{n-1}^{(1)}\left(\kappa_{\mathrm{p}} a\right) H_{n+1}^{(1)}\left(\kappa_{\mathrm{s}} a\right)+H_{n-1}^{(1)}\left(\kappa_{\mathrm{s}} a\right) H_{n+1}^{(1)}\left(\kappa_{\mathrm{p}} a\right)\right] .
$$

Next we shall derive more explicit expressions of the leading terms $\phi_{0}^{(n)}(r), \psi_{0}^{(n)}(r)$ and the linear terms $\phi_{1}^{(n)}(r), \psi_{1}^{(n)}(r)$, which make it possible to obtain an explicit reconstruction formula for the inverse problem.

3.4. Leading terms. For $k=0$ it follows from (3.7)-(3.9) that

$$
v_{0}=w_{0}=p_{0}=q_{0}=0, \quad \sigma_{0}=\rho_{\mathrm{p}}, \quad \tau_{0}=\rho_{\mathrm{s}} .
$$

Recalling (2.16), we obtain the Fourier coefficients:

$$
v_{0}^{(n)}=w_{0}^{(n)}=p_{0}^{(n)}=q_{0}^{(n)}=0, \quad \sigma_{0}^{(n)}=\alpha_{n}^{(2)} W(b) / H_{n}^{(1)}\left(\kappa_{\mathrm{p}} b\right), \quad \tau_{0}^{(n)}=\beta_{n}^{(2)} W(b) / H_{n}^{(1)}\left(\kappa_{\mathrm{s}} b\right) .
$$

Substituting the above quantities into (3.13) and noting (2.15) yields

$$
\zeta_{0}^{(n)}=-b W(b) \alpha_{n}^{(2)}, \quad \eta_{0}^{(n)}=-b W(b) \beta_{n}^{(2)} .
$$

Plugging the above quantities into (3.14), we get

$$
\left\{\begin{array}{l}
\phi_{0}^{(n)}(a)=-b W(b) \Lambda_{n}^{-1}\left[\kappa_{\mathrm{s}} a H_{n}^{(1)^{\prime}}\left(\kappa_{\mathrm{s}} a\right) \alpha_{n}^{(2)}-\mathrm{i} n H_{n}^{(1)}\left(\kappa_{\mathrm{p}} a\right) \beta_{n}^{(2)}\right] \\
\psi_{0}^{(n)}(a)=-b W(b) \Lambda_{n}^{-1}\left[\kappa_{\mathrm{p}} a H_{n}^{(1)^{\prime}}\left(\kappa_{\mathrm{p}} a\right) \beta_{n}^{(2)}+\mathrm{i} n H_{n}^{(1)}\left(\kappa_{\mathrm{s}} a\right) \alpha_{n}^{(2)}\right] .
\end{array}\right.
$$


Substituting (3.15), (3.16) into (3.12) and noting that

$$
K^{(n)}(r, a ; \kappa)=-H_{n}^{(1)}(\kappa r) /\left(\kappa H_{n}^{(1)^{\prime}}(\kappa a)\right), \quad K^{(n)}(r, b ; \kappa)=H_{n}^{(1)}(\kappa b) R_{n}(r ; \kappa) / W(b),
$$

we obtain explicit expressions of the leading terms:

$$
\left\{\begin{array}{l}
\phi_{0}^{(n)}(r)=-\frac{b W(b)}{\Lambda_{n}}\left[\frac{n^{2} H_{n}^{(1)}\left(\kappa_{\mathrm{s}} a\right)}{\kappa_{\mathrm{p}} a H_{n}^{(1)^{\prime}}\left(\kappa_{\mathrm{p}} a\right)} \alpha_{n}^{(2)}-\mathrm{i} n \beta_{n}^{(2)}\right] H_{n}^{(1)}\left(\kappa_{\mathrm{p}} r\right)+\alpha_{n}^{(2)} R_{n}\left(r ; \kappa_{\mathrm{p}}\right), \\
\psi_{0}^{(n)}(r)=-\frac{b W(b)}{\Lambda_{n}}\left[\frac{n^{2} H_{n}^{(1)}\left(\kappa_{\mathrm{p}} a\right)}{\kappa_{\mathrm{s}} a H_{n}^{(1)^{\prime}}\left(\kappa_{\mathrm{s}} a\right)} \beta_{n}^{(2)}+\mathrm{i} n \alpha_{n}^{(2)}\right] H_{n}^{(1)}\left(\kappa_{\mathrm{s}} r\right)+\beta_{n}^{(2)} R_{n}\left(r ; \kappa_{\mathrm{s}}\right),
\end{array}\right.
$$

where $R_{n}$ is given in (A.3).

3.5. Linear terms. For $k=1$ it follows from (3.7) that

$$
\left\{\begin{array}{c}
v_{1}=h^{-1} r^{-2}\left\{2 r(r-b) g \partial_{r r}+\left[(2 r-b) g-(r-b) g^{\prime \prime}\right] \partial_{r}+2 g \partial_{\theta \theta}\right. \\
\left.-2(r-b) g^{\prime} \partial_{r \theta}+2 r(2 r-b) g \kappa_{p}^{2}\right\} \phi_{0}(r, \theta) \\
w_{1}=h^{-1} r^{-2}\left\{2 r(r-b) g \partial_{r r}+\left[(2 r-b) g-(r-b) g^{\prime \prime}\right] \partial_{r}+2 g \partial_{\theta \theta}\right. \\
\left.-2(r-b) g^{\prime} \partial_{r \theta}+2 r(2 r-b) g \kappa_{s}^{2}\right\} \psi_{0}(r, \theta)
\end{array}\right.
$$

Using the Fourier series expansion and the convolution theorem yields

$$
v_{1}^{(n)}(r)=\sum_{m \in \mathbb{Z}} V_{n, m}(r) g^{(n-m)}, \quad w_{1}^{(n)}(r)=\sum_{m \in \mathbb{Z}} W_{n, m}(r) g^{(n-m)},
$$

where

$$
\left\{\begin{array}{c}
V_{n, m}(r)=h^{-1} r^{-2}\left\{2 r(r-b) \partial_{r r}+\left[(2 r-b)+\left(n^{2}-m^{2}\right)(r-b)\right] \partial_{r}\right. \\
\left.+\left[2 r(2 r-b) \kappa_{p}^{2}-2 m^{2}\right]\right\} \phi_{0}^{(m)}(r), \\
W_{n, m}(r)=h^{-1} r^{-2}\left\{2 r(r-b) \partial_{r r}+\left[(2 r-b)+\left(n^{2}-m^{2}\right)(r-b)\right] \partial_{r}\right. \\
\left.+\left[2 r(2 r-b) \kappa_{s}^{2}-2 m^{2}\right]\right\} \psi_{0}^{(m)}(r),
\end{array}\right.
$$

By (3.8) we have

$$
\left\{\begin{array}{l}
p_{1}=-a^{-1}\left[g\left(\partial_{r} \phi_{0}-h^{-1} \partial_{\theta} \psi_{0}\right)-g^{\prime} \partial_{r} \psi_{0}\right] \\
q_{1}=-a^{-1}\left[g\left(\partial_{r} \psi_{0}+h^{-1} \partial_{\theta} \phi_{0}\right)+g^{\prime} \partial_{r} \phi_{0}\right]
\end{array}\right.
$$

Their Fourier coefficients are given by

$$
p_{1}^{(n)}=\sum_{m \in \mathbb{Z}} A_{n, m} g^{(n-m)}, \quad q_{1}^{(n)}=\sum_{m \in \mathbb{Z}} B_{n, m} g^{(n-m)}
$$

where

$$
\left\{\begin{array}{l}
A_{n, m}=-a^{-1}\left[\partial_{r} \phi_{0}^{(m)}-\mathrm{i} m h^{-1} \psi_{0}^{(m)}-\mathrm{i}(n-m) \partial_{r} \psi_{0}^{(m)}\right] \\
B_{n, m}=-a^{-1}\left[\partial_{r} \psi_{0}^{(m)}+\mathrm{i} m h^{-1} \phi_{0}^{(m)}+\mathrm{i}(n-m) \partial_{r} \phi_{0}^{(m)}\right] .
\end{array}\right.
$$

By (3.9) we obtain

$$
\sigma_{1}=-h^{-1} g\left(\mathscr{T}_{\mathrm{p}} \phi_{0}+\rho_{\mathrm{p}}\right), \quad \tau_{1}=-h^{-1} g\left(\mathscr{T}_{\mathrm{s}} \psi_{0}+\rho_{\mathrm{s}}\right) .
$$

Their Fourier coefficients are given by

$$
\sigma_{1}^{(n)}=\sum_{m \in \mathbb{Z}} S_{m} g^{(n-m)}, \quad \tau_{1}^{(n)}=\sum_{m \in \mathbb{Z}} T_{m} g^{(n-m)},
$$


where

$$
S_{m}=-h^{-1}\left[\gamma_{\mathrm{p}}^{(m)} \phi_{0}^{(m)}+\rho_{\mathrm{p}}^{(m)}\right], \quad T_{m}=-h^{-1}\left[\gamma_{\mathrm{s}}^{(m)} \psi_{0}^{(m)}+\rho_{\mathrm{s}}^{(m)}\right] .
$$

Substituting (3.18)-(3.20) into (3.13) yields

$$
\zeta_{1}^{(n)}=\sum_{m \in \mathbb{Z}} X_{n, m} g^{(n-m)}, \quad \eta_{1}^{(n)}=\sum_{m \in \mathbb{Z}} Y_{n, m} g^{(n-m)},
$$

where

$$
\left\{\begin{array}{l}
X_{n, m}=a H_{n}^{(1)}\left(\kappa_{\mathrm{p}} a\right) A_{n, m}-b H_{n}^{(1)}\left(\kappa_{\mathrm{p}} b\right) S_{m}+\int_{a}^{b} s H_{n}^{(1)}\left(\kappa_{\mathrm{p}} s\right) V_{n, m}(s) \mathrm{d} s \\
Y_{n, m}=a H_{n}^{(1)}\left(\kappa_{\mathrm{s}} a\right) B_{n, m}-b H_{n}^{(1)}\left(\kappa_{\mathrm{s}} b\right) T_{m}+\int_{a}^{b} s H_{n}^{(1)}\left(\kappa_{\mathrm{s}} s\right) W_{n, m}(s) \mathrm{d} s
\end{array}\right.
$$

Plugging (3.21) into (3.14) leads to

$$
\phi_{1}^{(n)}(a)=\sum_{m \in \mathbb{Z}} C_{n, m} g^{(n-m)}, \quad \psi_{1}^{(n)}(a)=\sum_{m \in \mathbb{Z}} D_{n, m} g^{(n-m)},
$$

where

$$
\left\{\begin{array}{l}
C_{n, m}=\Lambda_{n}^{-1}\left[\kappa_{\mathrm{s}} a H_{n}^{(1)^{\prime}}\left(\kappa_{\mathrm{s}} a\right) X_{n, m}-\mathrm{i} n H_{n}^{(1)}\left(\kappa_{\mathrm{p}} a\right) Y_{n, m}\right] \\
D_{n, m}=\Lambda_{n}^{-1}\left[\kappa_{\mathrm{p}} a H_{n}^{(1)^{\prime}}\left(\kappa_{\mathrm{p}} a\right) Y_{n, m}+\mathrm{i} n H_{n}^{(1)}\left(\kappa_{\mathrm{s}} a\right) X_{n, m}\right] .
\end{array}\right.
$$

Substituting (3.18)-(3.20) and (3.22) into (3.12), evaluating at $r=b$, and noting that

$$
\left\{\begin{array}{l}
K^{(n)}(b, a ; \kappa)=-H_{n}^{(1)}(\kappa b) /\left(\kappa H_{n}^{(1)^{\prime}}(\kappa a)\right) \\
K^{(n)}(b, b ; \kappa)=H_{n}^{(1)}(\kappa b) R_{n}(b ; \kappa) / W(b) \\
K^{(n)}(b, s ; \kappa)=H_{n}^{(1)}(\kappa b) R_{n}(s ; \kappa) / W(s)
\end{array}\right.
$$

we obtain explicit expressions of the leading terms at $r=b$ :

$$
\phi_{1}^{(n)}(b)=\sum_{m \in \mathbb{Z}} P_{n, m} g^{(n-m)}, \quad \psi_{1}^{(n)}(b)=\sum_{m \in \mathbb{Z}} Q_{n, m} g^{(n-m)},
$$

where

$$
\left\{\begin{array}{l}
P_{n, m}=H_{n}^{(1)}\left(\kappa_{\mathrm{p}} b\right)\left(\frac{A_{n, m}-\mathrm{i} n a^{-1} D_{n, m}}{\kappa_{\mathrm{p}} H_{n}^{(1)^{\prime}}\left(\kappa_{\mathrm{p}} a\right)}+\frac{R_{n}\left(b ; \kappa_{\mathrm{p}}\right)}{W(b)} S_{m}-\int_{a}^{b} \frac{R_{n}\left(s ; \kappa_{\mathrm{p}}\right)}{W(s)} V_{n, m}(s) \mathrm{d} s\right), \\
Q_{n, m}=H_{n}^{(1)}\left(\kappa_{\mathrm{s}} b\right)\left(\frac{B_{n, m}+\mathrm{i} n a^{-1} C_{n, m}}{\kappa_{\mathrm{s}} H_{n}^{(1)^{\prime}}\left(\kappa_{\mathrm{s}} a\right)}+\frac{R_{n}\left(b ; \kappa_{\mathrm{s}}\right)}{W(b)} T_{m}-\int_{a}^{b} \frac{R_{n}\left(s ; \kappa_{\mathrm{s}}\right)}{W(s)} W_{n, m}(s) \mathrm{d} s\right) .
\end{array}\right.
$$

It is clear to note the relation of the Fourier coefficients between $\phi_{1}, \psi_{1}$ and $g$ in (3.23) that the linear terms of the wave field contain all the information about the surface function. This observation motivates us to design an explicit inversion formula to reconstruct the surface function.

\section{INVERSE SCATTERING}

In this section, we introduce an explicit reconstruction formula and a nonlinear correction algorithm to improve the accuracy of the reconstruction. 
4.1. Reconstruction formula. Let $\boldsymbol{u}=\left[u_{1}, u_{2}\right]$. By the Helmholtz decomposition (2.18) we have

$$
u_{1}=\partial_{x} \phi+\partial_{y} \psi, \quad u_{2}=-\partial_{x} \psi+\partial_{y} \phi
$$

which can be equivalently written in the polar coordinate:

$$
\left\{\begin{array}{l}
u_{1}=\left[(\cos \theta) \partial_{r}-b^{-1}(\sin \theta) \partial_{\theta}\right] \phi+\left[(\sin \theta) \partial_{r}+b^{-1}(\cos \theta) \partial_{\theta}\right] \psi, \\
u_{2}=\left[-(\cos \theta) \partial_{r}+b^{-1}(\sin \theta) \partial_{\theta}\right] \psi+\left[(\sin \theta) \partial_{r}+b^{-1}(\cos \theta) \partial_{\theta}\right] \phi
\end{array}\right.
$$

Each of the above equation can be used independently to derive a reconstruction formula for the given data $\boldsymbol{u}$ on $\Gamma_{b}$. However, a more convenient formula can be obtained by considering the following combination of the data. Let

$$
v=(\cos \theta) u_{1}+(\sin \theta) u_{2}=\partial_{r} \phi+b^{-1} \partial_{\theta} \psi \quad \text { on } \Gamma_{b},
$$

which is equivalent to

$$
v=\mathscr{T}_{\mathrm{p}} \phi+b^{-1} \partial_{\theta} \psi+\rho_{\mathrm{p}} \quad \text { on } \Gamma_{b} .
$$

Considering the power series expansion

$$
v(x, y ; \varepsilon)=\sum_{m=0}^{\infty} v_{m}(x, y) \varepsilon^{m} .
$$

We apply the Fourier series expansions to (4.1) and obtain the Fourier coefficients for the leading term:

$$
v_{0}^{(n)}(b)=\gamma_{\mathrm{p}}^{(n)} \phi_{0}^{(n)}(b)+\mathrm{i} n b^{-1} \psi_{0}^{(n)}(b)+\rho_{\mathrm{p}}^{(n)},
$$

where $\phi_{0}^{(n)}$ and $\psi_{0}^{(n)}$ are given by (3.17). Using (3.23) we find the Fourier coefficients for the linear term

$$
v_{1}^{(n)}(b)=\sum_{m \in \mathbb{Z}} M(n, m) g^{(n-m)},
$$

where

$$
M(n, m)=\gamma_{\mathrm{p}}^{(n)} P_{n, m}+\mathrm{i} n b^{-1} Q_{n, m} .
$$

Now assume the measured data is given by $\boldsymbol{u}_{\delta}=\left[u_{1, \delta}, u_{2, \delta}\right]$ with

$$
u_{j, \delta}(b, \theta)=u_{j}(b, \theta)+\mathcal{O}(\delta), \quad j=1,2,
$$

where $u_{j}(b, \theta)$ denotes the noise-free data and $\delta$ denotes the noise level. Let

$$
v_{\delta}(b, \theta)=(\cos \theta) u_{1, \delta}(b, \theta)+(\sin \theta) u_{2, \delta}(b, \theta)
$$

be the combined noisy data. Clearly we have

$$
v_{\delta}(b, \theta)=v(b, \theta)+\mathcal{O}(\delta) .
$$

It follows from the power series expansion of $v$ that

$$
v_{\delta}(b, \theta)=v_{0}(b, \theta)+\varepsilon v_{1}(b, \theta)+\mathcal{O}\left(\varepsilon^{2}\right)+\mathcal{O}(\delta) .
$$

Dropping the high order remainder and the noise terms linearizes the inverse problem and leads to

$$
v_{\delta}(b, \theta)-v_{0}(b, \theta)=\varepsilon v_{1}(b, \theta) .
$$

Applying the Fourier series expansion, substituting (4.2), and noting the fact that $f=\varepsilon g$, we obtain the reconstruction formula in terms of the Fourier coefficients of the data and solution:

$$
v_{\delta}^{(n)}(b)-v_{0}^{(n)}(b)=\sum_{m \in \mathbb{Z}} M(n, m) f^{(n-m)} .
$$

It is clear to note that (4.4) is an infinite dimensional linear system of equations relating the Fourier coefficients of the surface profile $f$ and the data $v_{\delta}$. Hence we may obtain the Fourier coefficients of $f$ by inverting the above system of equations. In practice, it is necessary to truncate 
the infinite dimensional system to a finite dimensional system. Keeping only $f^{(m)}$ for $|m| \leq N$ in (4.4), we obtain a truncated finite dimensional linear system:

$$
L F=V_{\delta}-V_{0},
$$

where $L$ is the $(2 N+1) \times(2 N+1)$ matrix defined as

$$
L(m, n)=M(m, m-n), \quad-N \leq m, n \leq N,
$$

and $F, V_{\delta}, V_{0}$ are $(2 N+1) \times 1$ vectors defined as

$$
F(m)=f^{(m)}, \quad V_{\delta}(n)=v_{\delta}^{(n)}(b), \quad V_{0}(n)=v_{0}^{(n)}(b), \quad-N \leq m, n \leq N .
$$

Let

$$
F=L^{+}\left(V_{\delta}-V_{0}\right),
$$

where $L^{+}$denotes the Moore-Penrose pseudo-inverse of $L$. Then the reconstructed obstacle surface function is obtained as

$$
f=\sum_{|m| \leq N_{c}} F(m) e^{\mathrm{i} m \theta}
$$

where the spectral cut-off frequency $N_{c} \leq N$ may be determined from the signal-to-noise ratio.

We point out that the error for the above reconstruction formula consists of the following parts.

(1) regularization error by dropping high frequency modes of $f$;

(2) approximation error by truncating the infinite dimensional linear system (4.4) to the finite dimensional linear system (4.5);

(3) linearization error by dropping high order terms in the power series expansion of $u_{j}$;

(4) noise in the measured data.

The convergence of the power series and the error estimate of the inverse problem require a dedicated regularity analysis of the solution for the direct problem and will be reported in a separate work. We refer to [8] for a related work on near-field imaging of periodic surfaces for acoustic waves, which establishes the well-posedness, convergence, and error estimates for the underlying direct and inverse problems.

4.2. Nonlinear correction algorithm. The reconstruction obtained by (4.6) and (4.7) is an approximate solution of the linearized inverse problem. They may serve as a good initial guess for an iterative algorithm that can improve the solution if the surface deformation parameter may not be small.

Let $F_{0}$ be the initial Fourier coefficients obtained by (4.6) and $f_{0}$ be the initial reconstruction obtained by (4.7). Using $f_{0}$ as the surface function for the obstacle, we may solve the direct scattering problem and obtain the Fourier coefficients for data $v_{f_{0}}^{(n)}(b)$. It follows from the reconstruction formula that we have an approximate relation:

$$
L F_{0}=V_{f_{0}}-V_{0},
$$

where $V_{f_{0}}$ is a $(2 N+1) \times 1$ vector given by $V_{f_{0}}(n)=v_{f_{0}}^{(n)}(b)$. Subtracting the above equation from (4.5) yields

$$
L\left(F-F_{0}\right)=V_{\delta}-V_{f_{0}} .
$$

Inverting the above equation yields the updated Fourier coefficients

$$
F_{1}=F_{0}+L^{+}\left(V_{\delta}-V_{f_{0}}\right) .
$$

Then the updated reconstructed surface function is

$$
f_{1}=\sum_{|m| \leq N_{c}} F_{1}(m) e^{\mathrm{i} m \theta}
$$


Repeating the above procedure yields the nonlinear correction algorithm:

$$
F_{j+1}=F_{j}+L^{+}\left(V_{\delta}-V_{f_{j}}\right), \quad f_{j+1}=\sum_{|m| \leq N_{c}} F_{j+1}(m) e^{\mathrm{i} m \theta}, \quad j=0,1, \ldots,
$$

where $V_{f_{j}}$ is a $(2 N+1) \times 1$ vector given by $V_{f_{j}}(n)=v_{f_{j}}^{(n)}(b)$. Here $v_{f_{j}}^{(n)}(b)$ is the Fourier coefficient of the data field which is from the solution of the direct problem corresponding to the surface function $f_{j}$. Since the coefficient matrix $L$ remains unchanged during iterations, it is efficient to implement the algorithm.

\section{Numerical EXPERIMENTS}

In this section, we consider some representative surface functions, investigate the dependence of the reconstructions on various parameters, and present numerical experiments to show the effectiveness of our method.

The synthetic data is obtained from solving the direct scattering problem by using the finite element method with the perfectly matched layer technique. Once the total displacement field $\boldsymbol{u}$ is obtained at the mesh points on $\Gamma_{b}$, it is interpolated to uniformly distributed points on $\Gamma_{b}$ through a cubic spline interpolation. To test the stability, we add an amount of noise to the data:

$$
\boldsymbol{u}^{\delta}\left(b, \theta_{i}\right)=\boldsymbol{u}\left(b, \theta_{i}\right)\left(1+\delta \boldsymbol{r}_{i}\right),
$$

where $\delta$ indicates the noise level and $\boldsymbol{r}_{i}$ is a random vector with values drawn from the uniform distribution in $[-1,1]$. The combined data $v_{\delta}$ is then obtained through (4.3) and its Fourier coefficients are computed from the fast Fourier transform.

In the following numerical experiments, we fix the angular frequency $\omega=\pi$, and the Lamé constants $\lambda=2, \mu=1$. The corresponding pressure and shear wavelength are 4 and 2 respectively. The radius of the unperturbed obstacle is taken as $a=1$. Hence any fine details of the perturbed obstacle is within the subwavelength regime with respect to either pressure or shear wave.

5.1. Incident field. Let the exact surface function be given by $f(\theta)=\varepsilon g(\theta)$, where

$$
g(\theta)=\exp (\sin 5 t)-1.5 \text {. }
$$

Since our method is derived for an arbitrary time-harmonic incident field, let's investigate the effect of different incident fields on the reconstructions.

First consider a time-harmonic plane compressional wave propagating in the angle $\theta_{0}$. Specifically, we have

$$
\phi_{\text {in }}=e^{\mathrm{i} \kappa_{\mathrm{p}} r \cos \left(\theta-\theta_{0}\right)}, \quad \psi_{\text {in }}=0, \quad \boldsymbol{u}_{\text {in }}=\nabla \phi_{\text {in }}=\mathrm{i} \kappa_{\mathrm{p}} \phi_{\text {in }}\left[\begin{array}{c}
\cos \theta_{0} \\
\sin \theta_{0}
\end{array}\right],
$$

where $\phi_{\text {in }}$ has the following expansion into Hankel functions

$$
\phi_{\text {in }}=\sum_{n \in \mathbb{Z}} \mathrm{i}^{n} J_{n}\left(\kappa_{\mathrm{p}} r\right) e^{\mathrm{i} n\left(\theta-\theta_{0}\right)}=\sum_{n \in \mathbb{Z}}\left[\frac{\mathrm{i}^{n}}{2} e^{-\mathrm{i} n \theta_{0}} H_{n}^{(1)}\left(\kappa_{\mathrm{p}} r\right)+\frac{\mathrm{i}^{n}}{2} e^{-\mathrm{i} n \theta_{0}} H_{n}^{(2)}\left(\kappa_{\mathrm{p}} r\right)\right] e^{\mathrm{i} n \theta} .
$$

Recalling (2.14), we have

$$
\alpha_{n}^{(1)}=\alpha_{n}^{(2)}=\frac{\mathrm{i}^{n}}{2} e^{-\mathrm{i} n \theta_{0}}, \quad \beta_{n}^{(1)}=\beta_{n}^{(2)}=0 .
$$

Due to the linearity of the linearized inverse problem, it is simple to consider an incident field with multiple wave components. For instance, we may consider an incident field with two plane pressure wave components propagating in the angles $\theta_{0}$ and $\theta_{1}$, i.e.,

$$
\phi_{\text {in }}=e^{\mathrm{i} \kappa_{\mathrm{p}} r \cos \left(\theta-\theta_{0}\right)}+e^{\mathrm{i} \kappa_{\mathrm{p}} r \cos \left(\theta-\theta_{1}\right)}, \quad \psi_{\text {in }}=0 .
$$


Then we have

$$
\alpha_{n}^{(1)}=\alpha_{n}^{(2)}=\frac{\mathrm{i}^{n}}{2}\left(e^{-\mathrm{i} n \theta_{0}}+e^{-\mathrm{i} n \theta_{1}}\right), \quad \beta_{n}^{(1)}=\beta_{n}^{(2)}=0 .
$$

This is still considered as a single incident field since the two plane waves are sent simultaneously from two different directions. The measurement is only taken once at $\Gamma_{b}$.

Another commonly used incident field is the one generated from a point source. For example, a pressure incident field generated at the point $\left(r_{0}, \theta_{0}\right)$ can be represented by

$$
\phi_{\text {in }}=H_{0}^{(1)}\left(\kappa_{\mathrm{p}} \sqrt{r^{2}-2 r r_{0} \cos \left(\theta-\theta_{0}\right)+r_{0}^{2}}\right), \quad \psi_{\text {in }}=0,
$$

where $\left(r_{0}, \theta_{0}\right)$ is a source point in the exterior of the obstacle. It follows from (2.14) and the boundedness of $\phi_{\text {in }}$ at $r=0$ that $\alpha_{n}^{(1)}=\alpha_{n}^{(2)}$ for all $n \in \mathbb{Z}$. Consequently, we have

$$
\phi_{\text {in }}^{(n)}(b)=\alpha_{n}^{(1)} H_{n}^{(1)}\left(\kappa_{\mathrm{p}} b\right)+\alpha_{n}^{(2)} H_{n}^{(2)}\left(\kappa_{\mathrm{p}} b\right)=2 \alpha_{n}^{(1)} J_{n}\left(\kappa_{\mathrm{p}} b\right),
$$

which implies

$$
\alpha_{n}^{(1)}=\alpha_{n}^{(2)}=\frac{\phi_{\text {in }}^{(n)}(b)}{2 J_{n}\left(\kappa_{\mathrm{p}} b\right)}, \quad \beta_{n}^{(1)}=\beta_{n}^{(2)}=0 .
$$

Again, multiple point sources may be used and the coefficients are simply the sum of the coefficients for each source.

We may also consider an incoming cylindrical pressure wave, i.e.,

$$
\phi_{\text {in }}=H_{2}^{(0)}\left(\kappa_{\mathrm{p}} r\right), \quad \psi_{\text {in }}=0, \quad \boldsymbol{u}_{\text {in }}=\nabla \phi_{\text {in }}=\kappa_{\mathrm{p}} H_{2}^{(0)^{\prime}}\left(\kappa_{\mathrm{p}} r\right)\left[\begin{array}{l}
x / r \\
y / r
\end{array}\right] .
$$

The corresponding coefficients are simply

$$
\alpha_{1}^{(n)}=0, \quad \alpha_{2}^{(n)}=\delta_{n 0}, \quad \beta_{1}^{(n)}=\beta_{2}^{(n)}=0,
$$

where $\delta$ is the Kronecker delta function. This type of incident field has been used in our previous work on inverse obstacle scattering for acoustic waves [20,22].

Let the deformation parameter $\varepsilon=0.1$, the measurement distance $h=0.2$, the noise level $\delta=1 \%$, and the truncation and cut-off frequencies $N=N_{c}=10$ be fixed. Figure 1 shows the reconstruction results using six different pressure incident fields. For the incident field propagating in one direction, we observe an excellent reconstruction on the illuminated side of the obstacle but a defect of the reconstruction on the shaded side of the obstacle. Comparing Figure 1(b) and Figure 1(c), we can see that the defect is severer if the point source is closer to the obstacle, which is an expected effect from the physical point of view. It should be pointed out this defect is milder for smaller obstacles and severer for larger ones. As shown in Figure 1(d), the defect can be completely removed by using the incoming cylindrical incident field, which accounts for an incident field illuminating from all the directions. Similar results can be achieved by using only two plane waves sent from opposite directions, or by using two point sources positioned at opposite sides of the obstacle, as shown in Figure 1(e) and Figure 1(f). In the rest of the numerical experiments, we will take the incident field to be two plane pressure waves propagating in the angles 0 and $\pi$ when investigating the effects of other parameters.

5.2. Deformation parameter. The explicit reconstruction formula is relied on the linearization of the inverse problem by dropping all the nonlinear terms in the power series expansion. The reconstruction error will increase as the deformation parameter $\varepsilon$ increases. To investigate this effect, we set $h=0.3$ and $\delta=0$ fixed and consider three different deformation parameters $\varepsilon=0.05,0.1,0.15$. Figure 2 shows the reconstruction results and displays the expected results. 

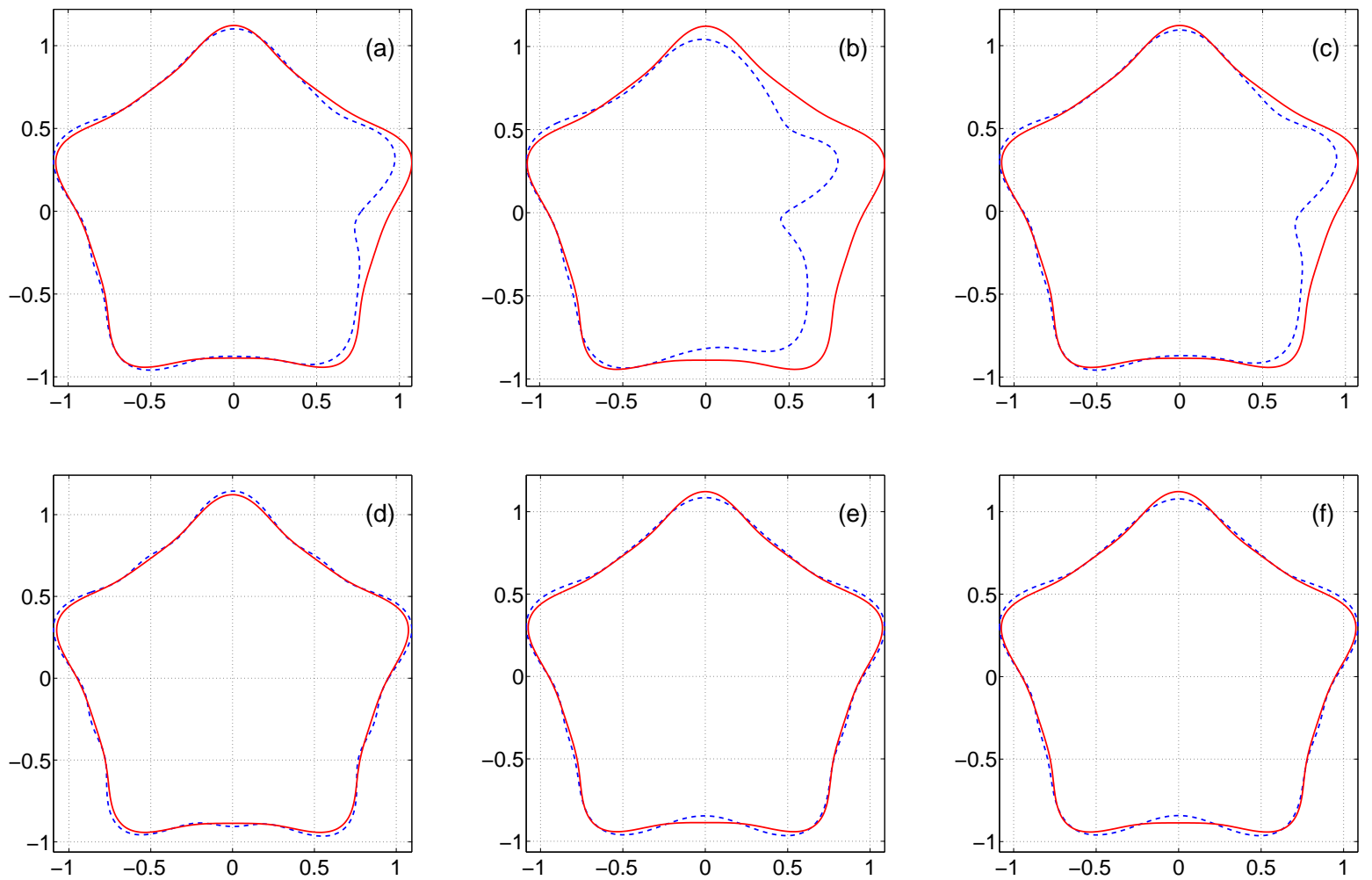

Figure 1. (Color online) Experiments of the incident field. The reconstructed obstacles (dashed lines) are plotted against the exact obstacles (solid lines) using the pressure incident field. (a) a plane wave from the left; (b) a point source at $(-2,0)$; (c) a point source at $(-10,0)$; (d) an incoming cylindrical wave; (e) two plane waves from the left and the right; (f) two point sources at $(-10,0)$ and $(10,0)$.
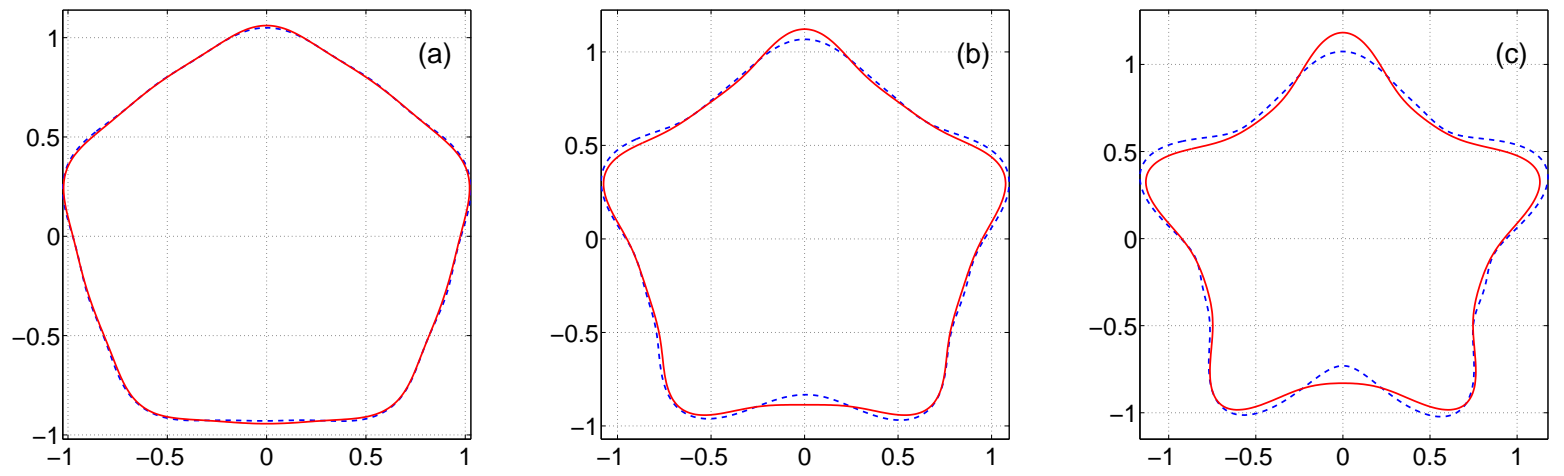

Figure 2. (Color online) Experiments of the deformation parameter $\varepsilon$. The reconstructed obstacles (dashed lines) are plotted against the exact obstacles (solid lines). (a) $\varepsilon=0.05$; (b) $\varepsilon=0.1$; (c) $\varepsilon=0.15$.

5.3. Nonlinear correction. An iterative algorithm is proposed in section 4.2 to improve accuracy for a large deformation parameter. To test this algorithm, we let $\varepsilon=0.15, h=0.3$ and $\delta=1 \%$ be fixed. Figure 3 shows the results by using the explicit reconstruction formula, the nonlinear correction 

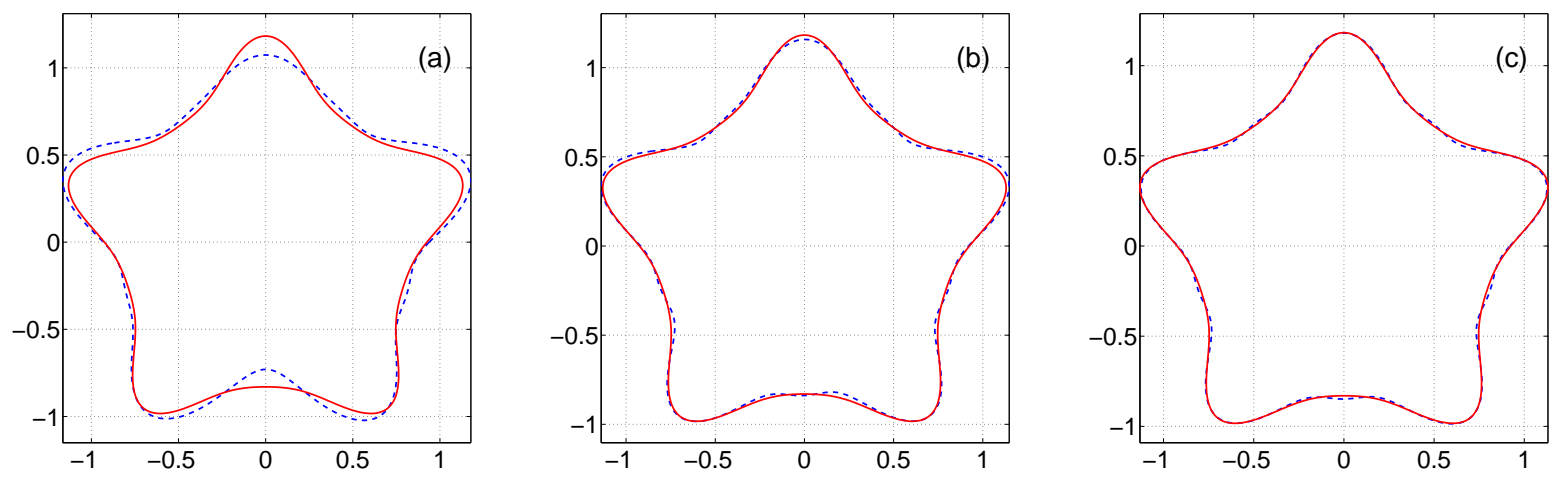

Figure 3. (Color online) Experiments of the nonlinear correction algorithm. The reconstructed obstacles (dashed lines) are plotted against the exact obstacles (solid line). (a) Explicit reconstruction formula with no iteration; (b) nonlinear correction with two iterations; (c) nonlinear correction with five iterations.
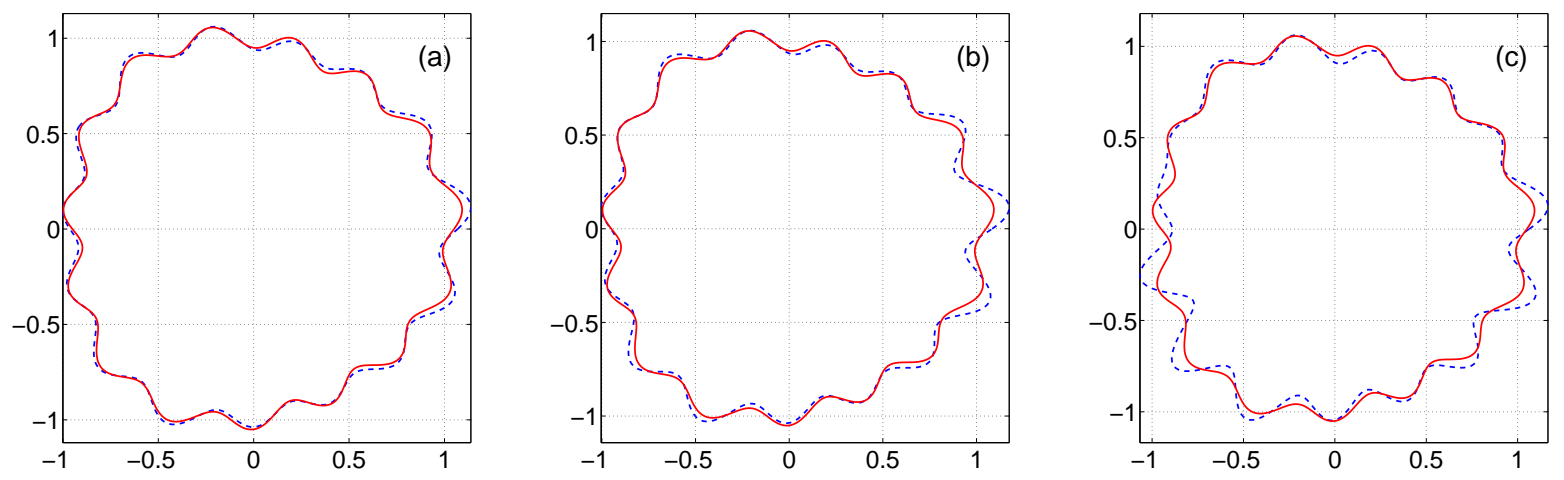

Figure 4. (Color online) Experiments of the measurement distance $h$. The reconstructed obstacles (dashed lines) are plotted against the exact obstacles (solid lines). (a) $h=0.2$; (b) $h=0.3$; (c) $h=0.4$.

with two iterations, and the nonlinear correction with five iterations, respectively. Evidently, the algorithm is very effective at improving the accuracy of the reconstruction in few number of iterations.

5.4. Measurement distance. Now we investigate the effect of the measurement distance $h$. We consider a different surface function. Let the exact surface function be given by $f(\theta)=\varepsilon g(\theta)$, where

$$
g(\theta)=0.5 \cos (3 \theta)+0.5 \sin (15 \theta) .
$$

This surface function displays more oscillation than the previous surface function in (5.1), although it contains only two nonzero Fourier modes. Figure 4 shows the reconstruction results with fixed $\varepsilon=0.1, \delta=5 \%$ and various $h=0.2,0.3,0.4$. It is clear to note that we have a better reconstruction when the measurement distance $h$ is smaller. This is consistent with the general principle of inverse scattering problems in near-field imaging.

5.5. Noise level. Next we consider the effect of noise in the measurement data. Figure 5 shows the results with three different noise levels $\delta=1 \%, 5 \%, 10 \%$ when fixing other parameters $\varepsilon=$ $0.1, h=0.2, N=N_{c}=15$. We observe that the reconstruction is very robust with respect to the measurement noise since the spectral cut-off regularization is adopted to suppress the possible amplified contamination of the noise. 

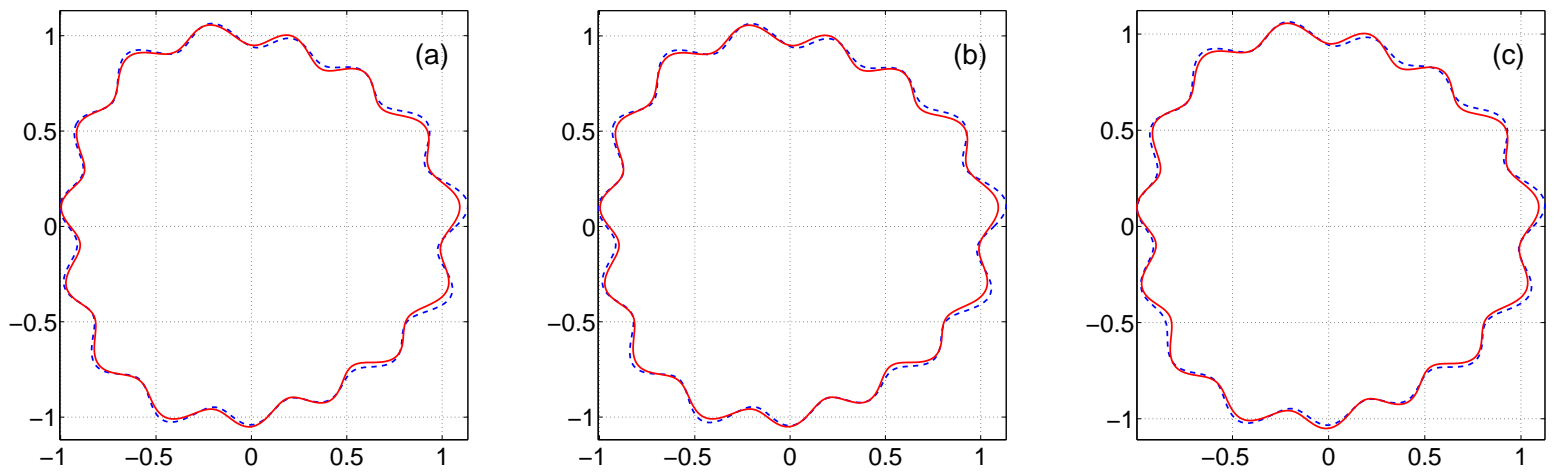

Figure 5. (Color online) Experiments of the noise level $\delta$. The reconstructed obstacles (dashed lines) are plotted against the exact obstacles (solid lines). (a) $\delta=1 \%$;

(b) $\delta=5 \%$; (c) $\delta=10 \%$.
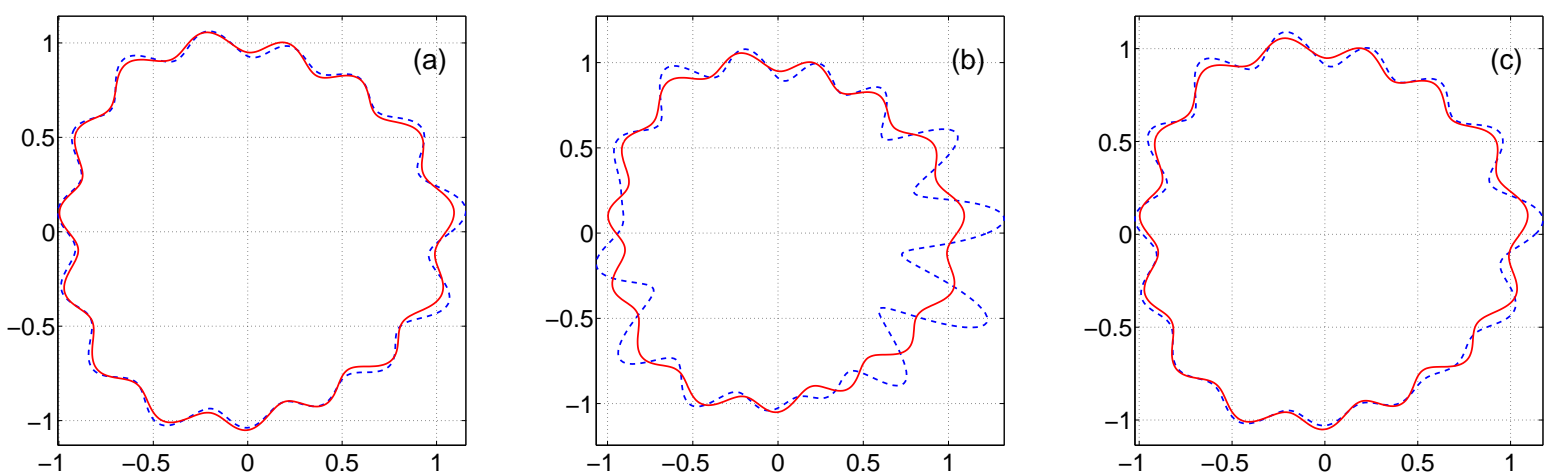

Figure 6. (Color online) Experiments of the number of measurement points. The reconstructed obstacles (dashed line) are plotted against the exact obstacles (solid line). (a) $\delta=0$ and 256 measurement points; (b) $\delta=20 \%$ and 256 measurement points; (c) $\delta=20 \%$ and 2560 measurement points.

5.6. Sample points. Another important factor that influences the reconstruction error due to the measurement noise is the number of measurement points. It is shown in [24] that the Fourier coefficients of an additive white noise has a variance of order $\mathcal{O}(1 / N)$, where $N$ is the number of samples. We have similar observations for the multiplicative noise used in our numerical experiments. As a consequence, the error due to the measurement noise can be made arbitrarily small by taking sufficiently large number of measurement points. Figure 6(a) shows the reconstruction result with no measurement noise and 256 measurement points, while Figure 6(b) shows the result with $20 \%$ noise and 256 measurement points. The other parameters are taken as $\varepsilon=0.1, h=0.3, N=N_{c}=15$. The influence of the noise is apparently seen from the results. However, if we increase the number of measurement points ten times to 2560, then we obtain the result in Figure 6(c). The influence of the noise is greatly reduced.

5.7. Cut-off frequency and non-smooth obstacles. Now we investigate the dependence of the reconstruction on the truncation frequency $N$ and the cut-off frequency $N_{c}$. Both parameters are used to reduce the infinitely dimensional problem to a finite dimensional problem. They can be considered as the regularization parameters controlling the trade-off between the fit of the data and stability of the solution. Regularization methods for inverse problems and the choice of regularization 

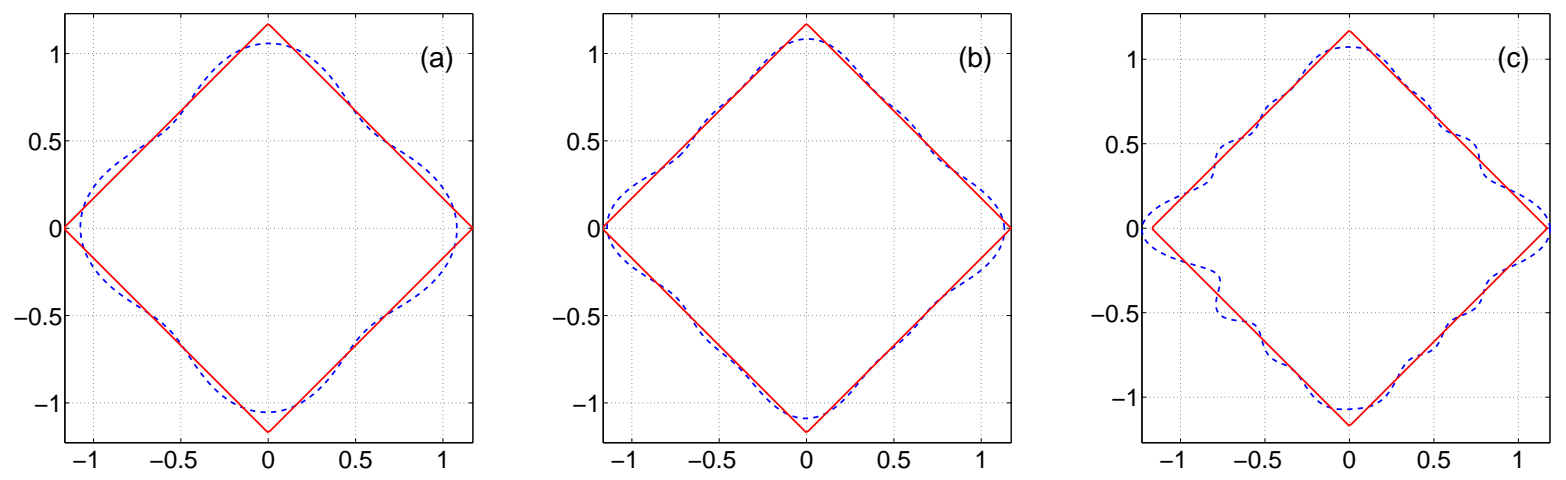

Figure 7. (Color online) Experiments of the cut-off frequency and a non-smooth surface function. The reconstructed obstacles (dashed lines) are plotted against the exact obstacles (solid lines). (a) $N=4$; (b) $N=8$; (c) $N=16$.

parameters are important research topics. We shall illustrate the influence of those parameters using a few examples for our problem. We refer to [12] and [19] for comprehensive treatment of those topics. It is possible to choose $N_{c}<N$ once $N$ is set. However, we find that there is no evident advantage for doing that so we always set $N_{c}=N$ in the numerical experiments.

Finally, we show that our method also works for non-smooth obstacles, although the mathematical judgment requires a smooth surface function. Let the boundary of the obstacle be a square. The size of the square is chosen in such a way that if $s$ is half of the side, then $\sqrt{2} s-a=a-s$, i.e., $s=2 a /(1+\sqrt{2})$. The corresponding deformation parameter is $\varepsilon=a-s=(\sqrt{2}-1) a /(\sqrt{2}-1) \approx 0.17$. Figure 7 shows the reconstruction results with $h=0.3, \delta=5 \%$ and $N=4,8,16$, respectively. Clearly the reconstructed obstacle becomes more oscillatory as $N$ increases and displays the well-known Gibbs phenomenon near the corners.

\section{Conclusion}

We presented an efficient method for solving the inverse obstacle scattering problem for elastic waves. By using the Helmholtz decomposition, we decomposed the displacement vector field into the compressional part and shear part, and derived a coupled system of boundary value problems. Utilizing the transformed field expansion, we reduced the two-dimensional problems into a successive sequence of coupled one-dimensional two-point boundary value problems, which were solved in closed forms. Assuming that the surface was a small and smooth perturbation of the circle and neglecting the nonlinear terms in the power series expansion, we linearized the inverse problem and deduced infinite dimensional linear systems relating the Fourier coefficients of the surface function and the data. The final reconstruction formula was obtained by truncating the systems to finite dimensions. We conducted comprehensive numerical experiments to demonstrate the effectiveness of the method and to investigate the dependence of the reconstruction on various parameters. The method requires a single incident field at a fixed frequency and is computationally efficient. The incident field can be a plane wave, point source or incoming cylindrical. The method works very well for smooth, oscillatory, and even non-smooth surfaces with super-resolved resolution. The proposed nonlinear correction algorithm is effective at improving accuracy of the reconstruction in few number of iterations.

The method should be readily modified to solve the transmission problems, where the background medium is not rigid and allows elastic waves to penetrate. It can also be extended to the threedimensional geometry without essential difficulties. A difficult and interesting problem is to consider elongated surfaces, such as a perturbation of an ellipse. More challenging problems include the convergence analysis of the proposed method, inverse scattering of random surfaces, and multiple obstacles. We hope to work on some of these problems and report the results in the near future. 


\section{Appendix A. A two-Point Boundary VAlue PROBlem}

Consider a two-point boundary value problem:

$$
\begin{cases}{\left[\partial_{r r}+r^{-1} \partial_{r}+\left(\kappa^{2}-n^{2} r^{-2}\right)\right] w=v,} & a<r<b, \\ \partial_{r} w=p, & r=a, \\ {\left[\partial_{r}-\kappa H_{n}^{(1)^{\prime}}(\kappa b) / H_{n}^{(1)}(\kappa b)\right] w=q,} & r=b .\end{cases}
$$

Lemma A.1. The two-point boundary value problem (A.1) has a unique solution given by

$$
w(r)=-K^{(n)}(r, a ; \kappa) p+K^{(n)}(r, b ; \kappa) q-\int_{a}^{b} K^{(n)}(r, s ; \kappa) v(s) \mathrm{d} s,
$$

where

$$
K^{(n)}(r, s ; \kappa)=\frac{1}{W(s)} \begin{cases}H_{n}^{(1)}(\kappa r) R_{n}(s ; \kappa), & s \leq r, \\ H_{n}^{(1)}(\kappa s) R_{n}(r ; \kappa), & s \geq r .\end{cases}
$$

Here $W$ is the Wronskian given in (2.15) and

$$
R_{n}(z ; \kappa)=H_{n}^{(2)}(\kappa z)-\frac{H_{n}^{(2)^{\prime}}(\kappa a)}{H_{n}^{(1)^{\prime}}(\kappa a)} H_{n}^{(1)}(\kappa z) .
$$

Proof. It follows from the variation of parameters that the differential equation (A.1) admits the general solution:

$$
w(r)=w_{1}(r)\left[c_{1}-\int_{a}^{r} \frac{w_{2}(s) v(s)}{W(s)} \mathrm{d} s\right]+w_{2}(r)\left[c_{2}+\int_{a}^{r} \frac{w_{1}(s) v(s)}{W(s)} \mathrm{d} s\right],
$$

where $w_{1}(r)=H_{n}^{(1)}(\kappa r)$ and $w_{2}(r)=H_{n}^{(2)}(\kappa r)$ are two linearly independent solutions for the corresponding homogeneous differential equation, $W$ is the Wronskian of $w_{1}, w_{2}$, and $c_{1}, c_{2}$ are constants to be determined from the boundary conditions. Substituting (A.4) into the boundary conditions (A.1) yields

$$
c_{1}=\frac{p}{u_{1}^{\prime}(a)}-\frac{u_{2}^{\prime}(a)}{u_{1}^{\prime}(a)} c_{2}, \quad c_{2}=\frac{u_{1}(b)}{W(b)} q-\int_{a}^{b} \frac{u_{1}(s) v(s)}{W(s)} \mathrm{d} s .
$$

The proof is completed by plugging (A.5) into (A.4).

We notice that the function $R_{n}(z ; \kappa)$ in (A.3) is well-defined for all $z>0$ since $H_{n}^{(1)^{\prime}}(z) \neq 0$ for all $z>0$. It is also useful to note that

$$
R_{n}(a ; \kappa)=-W(\kappa a) / H_{n}^{(1)^{\prime}}(\kappa a)=-W(a) /\left(\kappa H_{n}^{(1)^{\prime}}(\kappa a)\right) .
$$

\section{REFERENCES}

[1] H. Ammari, E. Beretta, E. Francini, H. Kang, M. Lim, Optimization algorithm for reconstructing interface changes of a conductivity inclusion from modal measurements, Math. Comp., 79 (2010) 1757-1777.

[2] H. Ammari, E. Beretta, E. Francini, H. Kang and M. Lim Reconstruction of small interface changes of an inclusion from modal measurements II: the elastic case. J. Math. Pure. Appl. 94 (2010), 322-339.

[3] H. Ammari, H. Kang, M. Lim, and H. Zribi, Conductivity interface problems. Part I: small perturbations of an interface, Trans. Amer. Math. Soc. 362 (2010), 2435-2449.

[4] T. Arens, Linear sampling methods for 2D inverse elastic wave scattering, Inverse Problems, 17 (2001).

[5] G. Bao, T. Cui, and P. Li, Inverse diffraction grating of Maxwell's equations in biperiodic structures, Optics Express, 22 (2014), 4799-4816.

[6] G. Bao and P. Li, Near-field imaging of infinite rough surfaces, SIAM J. Appl. Math., 73 (2013), $2162-2187$.

[7] G. Bao and P. Li, Near-field imaging of infinite rough surfaces in dielectric media, SIAM J. Imaging Sci., 7 (2014), $867-889$.

[8] G. Bao and P. Li, Convergence analysis in near-field imaging, Inverse Problems, 30 (2014), 085008. 
[9] A. Charalambopoulos, A. Kirsch, K. A. Anagnostopoulos, D. Gintides and K. Kiriaki, The factorization method in inverse elastic scattering from penetrable bodies, Inverse Problems 23 (2007), 27-51.

[10] T. Cheng, P. Li, and Y. Wang, Near-field imaging of perfectly conducting grating surfaces, J. Opt. Soc. Am. A, 30 (2013), 2473-2481.

[11] D. Courjon, Near-Field Microscopy and Near-Field Optics, Imperial College, 2003.

[12] H. W. Engl, M. Hanke, and A. Neubauer, Regularization of Inverse Problems, Springer, 1996.

[13] S. N. Fata and B. B. Guzina, A linear sampling method for near-field inverse problems in elastodynamics, Inverse Problems 20 (2004), 713-736.

[14] D. Gintides and M. Sini, Identification of obstacles using only the scattered P-waves or the scattered S-waves, Inverse Probl. Imag. 6 (2012), 39-55.

[15] C. Girard and A. Dereux, Near-field optics theories, Rep. Prog. Phys. 59, 657-699 (1996).

[16] G. Hu, A. Kirsch and M. Sini, Some inverse problems arising from elastic scattering by rigid obstacles, Inverse Problems 29 (2013), 015009.

[17] M. Kar and M. Sini, Reconstruction of interfaces from the elastic farfield measurements using CGO solutions, SIAM J. Math. Anal., 46 (2014), 2650-2691.

[18] M. Kar and M. Sini, On the inverse elastic scattering by interfaces using one type of scattered waves, J. Elasticity 118 (2015), 15-38.

[19] A. Kirsch, An Introduction to the Mathematical Theory of Inverse Problems, Springer, 2011.

[20] P. Li and Y. Wang, Near-field imaging of obstacles, Inverse Probl. Imag., 9 (2015), 189-210.

[21] P. Li and Y. Wang, Near-field imaging of interior cavities, Commun. Comput. Phys., 17 (2015), 542-563.

[22] P. Li and Y. Wang, Numerical solution of an inverse obstacle scattering problem with near-field data, J. Comput. Phys., 290 (2015), 157-168.

[23] P. Li, Y. Wang and Y. Zhao, Inverse elastic surface scattering with near-field data, Inverse Problems, 31 (2015), 035009.

[24] J. Schoukens and J. Renneboog, Modeling the noise influence on the Fourier coefficients after a discrete Fourier transform, IEEE Trans. Instrum. Meas., 35 (1986) 278-286.

Department of Mathematics, Purdue University, West Lafayette, IN 47907, USA.

E-mail address: lipeijun@math.purdue.edu

Department of Mathematics, Purdue University, West Lafayette, IN 47907, USA.

E-mail address: wang2049@math.purdue.edu 